\title{
Chaotic principle: an experimental test
}

\author{
F. Bonetto*, G. Gallavotti!, P. L. Garrido ${ }^{\circledR}$ \\ * Matematica, I ${ }^{a}$ Università di Roma, P.le Moro 2, 00185 Roma, Italia \\ 'Fisica, I ${ }^{a}$ Università di Roma, P.le Moro 2, 00185 Roma, Italia \\ ${ }^{\circledR}$ Instituto Carlos I de Física Teórica y Computacional, Universidad de Granada \\ E-18071 Granada, España
}

\begin{abstract}
The chaotic hypothesis discussed in [GC1] is tested experimentally in a simple conduction model. Besides a confirmation of the hypothesis predictions the results suggest the validity of the hypothesis in the much wider context in which, as the forcing strength grows, the attractor ceases to be an Anosov system and becomes an Axiom A attractor. A first test of the new predictions is also attempted.
\end{abstract}

Keywords: Chaotic hypothesis, Reversibility, Entropy, Dynamical systems

\section{§1. Introduction.}

A principle holding when motions have an empirically chaotic nature was introduced in reference [GC2]:

Chaotic hypothesis: A many particle system in a stationary state can be regarded as a smooth dynamical system with a transitive ${ }^{1}$ Axiom A global attractor for the purpose of computing macroscopic properties. In the reversible case it can be regarded, for the same purposes, as a smooth transitive Anosov system.

For an informal discussion of the properties of Anosov systems relevant for this work, in particular for the "Boltzmanian representation" of the SRB distribution possible for them, see [GC2], [G1]. See [AA], [Sm] for a general discussion of the basic geometrical ideas and $[\mathrm{S}],[\mathrm{R} 1],[\mathrm{R} 2],[\mathrm{Bo}]$ for the original and complete descriptions of the mathematical notion and properties of Anosov and Axiom A systems.

The results of this work mainly concern the reversible Anosov case: in the concluding remarks we discuss various questions related to reversibility and to the Axiom A cases. Therefore the part of the hypothesis that concerns reversible systems is essential for our applications. It can be rephrased in various ways and by doing so one gains some insights into the meaning of it: see $\S 6$.

This implies that the macroscopic time averages are described by a probability distribution $\mu$ on the "phase space" $\mathcal{C}$ of observed events, also called timing events, (which could be, for instance, the occurrence of a microscopic binary "collision"). The time evolution, or the $d y$ namics, is a map $S$ of $\mathcal{C}$ into itself. The map $S$ is derived from the flow $Q_{t}$ that solves the differential equations of motion of the system: the timing events $\mathcal{C}$ have to be thought as a surface transversal to the flow and if $t(x)$ is the time between the timing event $x$ and the successive one $S x$ it is $Q_{t(x)} x=S x$. Note that the points $Q_{t} x$ are not timing events (i.e. they are not in $\mathcal{C}$ ) for the intermediate times $0<t<t(x) .^{2}$ We call $Q_{t}$ the continuous time evolution

\footnotetext{
1 The notion of transitivity used in the above reference, and in the related ones, is that the stable and unstable manifolds of each point of the attractor are dense on the attractor.

2 One may wonder why we do not time the observations at constant pace: this would indeed be possible. It is however convenient to time the observations on natural events (i.e., using the jargon, "to make observations on a Poincaré's
} 
and $S$ the discrete time evolution.

The existence of the distribution $\mu$ is assumed in general, as stated by the following (extension) of the zeroth law, $[\mathrm{UF}]$, giving a global property of the motions generated by initial data chosen randomly with distribution $\mu_{0}$ proportional to the volume measure on $\mathcal{C}$ :

Extended zero-th law: A dynamical system $(\mathcal{C}, S)$ modeling a many particle system (or a continuum such as a fluid) describes motions that admit a statistics $\mu$ in the sense that, given any (smooth) macroscopic observable $F$ defined on the points $x$ of the phase space $\mathcal{C}$, the time average of $F$ exists for all $\mu_{0}$-randomly-chosen initial data $x$ and is given by:

$$
\lim _{M \rightarrow \infty} \frac{1}{M} \sum_{k=0}^{M-1} F\left(S^{j} x\right)=\int_{\mathcal{C}} \mu\left(d x^{\prime}\right) F\left(x^{\prime}\right) \stackrel{\text { def }}{=}\langle F\rangle_{+}
$$

where $\mu$ is a $S$-invariant probability distribution on $\mathcal{C}$.

The chaotic hypothesis was proposed by Ruelle in the case of fluid turbulence, and it is extended to non equilibrium many particle systems in [GC1]. If one assumes it, then it follows that the zeroth law holds, [S],[Bo],[R1]; however it is convenient to regard the two statements as distinct because the hypothesis we make is "only" that one can suppose that the system is Anosov for "practical purposes": this leaves the possibility that it is not strictly speaking such, and some corrections ("negligible in the thermodynamic limit") may be needed on the predictions obtained by using the hypothesis.

In [GC2] the generality of the hypothesis is discussed and in [GC1], [GC2], we derived, as a rather general consequence, predictions testable at least by numerical or physical experiments in systems with few degrees of freedom. The feature of the prediction relevant for numerical experiments, a large deviation theorem or fluctuation theorem, is that it is parameter free; other results concern the Onsager reciprocity in various classes of mechanical systems [G5], or fluid models [G4].

The theory was developed to understand the results in [ECM2] which, therefore, provided also the first test. In this paper we present the results of numerical experiments that we conducted in order to check the hypothesis in models different from the shear flow model in [ECM2].

Being a rather general principle, the chaotic hypothesis yields predictions that are sharp and inescapable, without free parameters. Hence it is important to check it with the highest precision possible. This immediately leads one to work at the limit of the present day computer capabilities and to lengthy data elaboration.

In $\S 2$ we describe the models and give a quantitative description of their rough characteristics, discussing the experiments that we perform. In $\S 3$ we explain, through an analogy with well known Ising model properties, the mechanism which allows us to acquire exact knowledge of some propeties of $\mu$ without actually computing $\mu$ itself (which might be a surprising "achievement" if one does not examine [GC1],[GC2] in some detail). In $\S 4$ we present the raw experimental results; in $\S 5$ and in the Appendix we briefly summarize the methods followed in statistical analysis of the result. In $\S 6$ we present comments, plans and perspectives, and some challenging pictures that seem to emerge from $[\mathrm{G} 4],[\mathrm{G} 5][\mathrm{BG}]$ and from the present paper.

section") so that one eliminates one degree of freedom as well as the corresponding (trivially zero) Lyapunov exponent, as recognized very early, [L]. 


\section{§2. The models and a description of the experiments.}

The models contain thermostat mechanisms in order to enable the systems to reach a non equilibrium stationary state in the presence of an imposed external field: therefore they are related to electrical conductivity problems. They represent a gas of $N$ identical particles with mass $m$, interacting via a hard core pair potential $\varphi$ with radius $r$ and with an external potential $\varphi^{e} \neq 0$. The gas is enclosed in a 2 dimensional box $\left[-\frac{k}{2} L, \frac{k}{2} L\right] \times\left[-\frac{\ell}{2} L, \frac{\ell}{2} L\right], k, \ell=1,2, \ldots$, and is subject to periodic boundary conditions and to a horizontal constant external field $E \underline{i}$ ( $\underline{i}$ is a unit vector in the $x$-direction). The external potential will also be just a hard core interaction excluding access to the area covered by some obstacles or forbidding the crossing of the box walls. The obstacles are hard disks with centers situated on two square lattices with spacing $L$ shifted by $\frac{1}{2} L$ relative to each other. The radii of the disks of each sub lattice are equal and respectively given by $R_{1}$ and $R_{2}$, so fixed that every rectilinear trajectory must suffer collisions with them. An alternative setting could have been a collection of identical hard disks (with large radii) with centers on a triangular lattice: the adopted geometry is the same as that of the previous paper [GG].

The geometry is very simple and the position space is described in Fig.1:

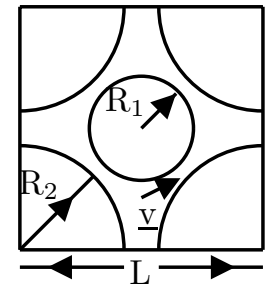

Fig.1: General billiard structure with scatterers of radius $R_{1}$ and $R_{2}$ in a periodic box with side length $L$, (case $k \times \ell=1 \times 1)$.

The box $\left[-\frac{k}{2} L, \frac{k}{2} L\right] \times\left[-\frac{\ell}{2} L, \frac{\ell}{2} L\right]$ consists of $k \cdot \ell$ unit lattice cells with side $L$ joined to form a square box: at the box boundary we impose periodic boundary conditions $(p b c)$ or, alternatively, semi periodic boundary conditions $\left(\frac{1}{2} p b c\right)$ : in the latter case the "horizontal" box walls are reflecting. The experiments with different boundary conditions have been performed "completely" independently, on different machines and with different codes.

The system is in contact with a "thermostat" which adds (or subtracts) energy so that the total internal energy stays rigorously constant. The equations of motion are:

$$
\begin{aligned}
& \underline{\dot{q}}_{j}=\frac{1}{m} \underline{p}_{j}, \quad \underline{\dot{p}}_{j}=\underline{F}_{j}+E \underline{i}-\alpha\left(\underline{p}_{)} \underline{p}_{j}\right. \\
& \underline{F}_{j} \equiv-\sum_{i \neq j} \underline{\partial}_{\underline{q}_{j}} \varphi\left(\underline{q}_{j}-\underline{q}_{i}\right)-\underline{\underline{\partial}}_{\underline{q}_{j}} \varphi^{e}\left(\underline{q}_{j}\right)
\end{aligned}
$$

with $j=1, \ldots, N ; \alpha(\underline{p})=E \underline{i} \cdot \sum_{j} \underline{p}_{j} /\left(\sum_{j} \underline{p}_{j}^{2}\right)$ and $\underline{F}_{j}$ is the (impulsive) force acting on particle $j$ (which is due only to the hard cores). The $\alpha$-term incorporates the coupling to a "Gaussian thermostat" and it is assumed to obey Gauss' "principle of least constraint", see [LA]. The constraint here is the constancy of the internal (i.e. kinetic) energy:

$$
H(\underline{p}, \underline{q})=\sum_{i=1}^{N} \frac{\underline{p}_{i}^{2}}{2 m} \stackrel{\text { def }}{=} e_{0} N
$$

a typical nonholonomic constraint; it follows then from Gauss' principle that the force corresponding to the constraint is proportional to the gradient with respect to $\underline{p}_{j}$ of $H$. This 
model has been studied in great detail, in [CELS], in the case $N=1$; a similar model has been investigated numerically in $[\mathrm{BEC}]$, and very recently in $[\mathrm{DPH}],[\mathrm{DM}]$. It is part of a wide class of models, see [GC1],[GC2], whose interest for the theory of non equilibrium stationary states was pointed out in $[\mathrm{HHP}],[\mathrm{PH}]$, where one can find the first studies performed in the context in which we are interested.

The timing events that we choose to follow are simply the collisions: whether with the walls or with the obstacles or with other particles. The boundary conditions will be periodic in both directions or reflecting in the one perpendicular to the field ("vertical") and periodic in the other ("horizontal").

The initial data will be fixed by a random choice with absolutely continuous distribution on the full phase space $\mathcal{F}$ (i.e. on the full energy surface).

The dimension of the phase space $\mathcal{F}$ of this system is that of the energy surface, i.e. $4 N-1$, and that of the set of timing events $\mathcal{C}$ is $2 D$ with $D=2 N-1$, i.e. one unit less than the dimension of $\mathcal{F}$. The phase space "contraction" rate, i.e. the divergence of the right hand side of $(2.2)$, is $\gamma(x)=D \alpha(x)$. It can be written in the form:

$$
\gamma(x)=D \alpha(x)=\frac{\varepsilon(x)}{k_{B} T(x)+\frac{2 N}{2 D} \frac{1}{2 m} \underline{\bar{p}}^{2}}
$$

where $\underline{\underline{p}}=\frac{1}{N} \sum_{j} \underline{p}$ is the average momentum, $\varepsilon(x)$ is the work done on the system per unit time by the external field and $\frac{1}{2} k_{B} T(x)$ is $\frac{1}{D} \sum_{j} \frac{1}{2 m}\left(\underline{p}_{j}-\underline{\bar{p}}\right)^{2}$ which, if $k_{B}$ is Boltzmann's constant, defines the kinetic temperature: hence the name of entropy production rate per (kinetic) degree of freedom that will be occasionally given to $\alpha(x)$. Note that $\gamma(x)$ does not have a definite sign. In dimension $d \geq 2$ the factor $\frac{2 N}{2 D}$ would become $\frac{2 N}{d D}$.

The above contraction corresponds to a contraction of the volume in the full phase space $\mathcal{F}$, between one collision and the next, given by $e^{-t_{0} \sigma(x)}$ with:

$$
\sigma(x)=\frac{1}{t_{0}} \int_{0}^{t(x)} \gamma\left(Q_{t} x\right) d t
$$

if $Q_{t}$ is the continuous time evolution (see $\S 1$ ) and $t_{0}$ denotes the mean collision time $t_{0}=\langle t(\cdot)\rangle_{+}$. We shall be concerned mostly with the contraction rate $\sigma_{\tau}(x)$ occurring during $\tau$ time steps as the system evolves between $S^{-\tau / 2} x$ and $S^{\tau / 2} x$ :

$$
\sigma_{\tau}(x) \stackrel{\text { def }}{=} \frac{1}{\tau} \sum_{j=-\tau / 2}^{\tau / 2-1} \sigma\left(S^{j} x\right)
$$

It has been proved, [CELS], that for $N=1$ and small $E \neq 0$ the average $\langle\sigma\rangle_{+}$is positive, i.e. the system is dissipative. There seems to be no reason to think that $\langle\sigma\rangle_{+}$is not positive when $N>1$ and our experiments show that indeed this seems to be the case.

Recently, in fact, it has been shown that under very general conditions (essentially under the assumption that the extended zero-th law holds) it must be $\langle\sigma\rangle_{+} \geq 0$, [R3]. In the latter paper it is also shown that $\langle\sigma\rangle_{+}>0$ if the $\mathrm{SRB}$ distribution $\mu$ gives probability 1 to a set which has zero Liouville measure (i.e. if the attractor is "really" smaller than the full phase space). ${ }^{3}$

Therefore it is natural to write:

3 We call attractor a set $G$ with minimal (Hausdorff) dimension which has the property that $\mu(G)=1$, i.e. which has probability 1 in the stationary state. In general the closure $\operatorname{clos}(G)$ of the attractor may be the whole space $\mathcal{C}$ while the dimension of $G$ may be much less. 


$$
\sigma_{\tau}(x)=\langle\sigma\rangle_{+} p(x)
$$

so that the contraction of the phase space volume, while the system evolves between $S^{-\tau / 2} x$ and $S^{\tau / 2} x$, is $e^{-\tau t_{0}\langle\sigma\rangle_{+} p(x)}$ and $\langle p\rangle_{+} \equiv 1$.

In this case the time reversal map $i$ defined by $i:(\underline{q}, \underline{p}) \rightarrow(\underline{q},-\underline{p})$ is such that $\sigma_{\tau}(i x)=$ $-\sigma_{\tau}(x)$.

The number $k \cdot l$ of unit lattice cells forming the box containing the gas will be called the size of the box. We define the density as $\rho=\frac{N}{k \ell L^{2}}$ and the energy density as $e_{0}=\frac{1}{N} \sum_{j} \frac{1}{2 m} \underline{p}_{j}^{2}$ and we take units so that $m=1, e_{0}=1 / 2, L=1$. The properties of the system are thus governed by the values of the parameters $R_{1}, R_{2}, r$ and $\rho$; in place of $\rho$ one could use the occupied volume $\delta=\frac{N V_{0}}{V-V_{o b s}}$ where $V_{0}$ is the particles volume, $V=k \ell L^{2}$ is the box volume and $V_{\text {obs }}$ is the total volume of the obstacles, so that $\delta$ is the ratio between the volume occupied by the particles cores and the free volume where they can roam (i.e. the volume of the cell outside the volume $V_{\text {obs }}$ occupied by the obstacles).

The field intensity $E$ is fixed to $E=1$ in all experiments. We shall consider systems with $N=2, N=10$, radii $R_{1}=0.2, R_{2}=0.4$ and $r=0.005$ or $r=0.01$ depending on the type of boundary conditions denoted, above, by $p b c$ and $\frac{1}{2} p b c$. Here is a list of the above kinematic quantities in the cases that we shall consider:

$\begin{array}{lll}\text { density } & \rho & \frac{N}{V} \\ \text { energy density } & e_{0} & \frac{1}{2} \\ \text { mass } & m & 1 \\ \text { box side } & L & 1 \\ \text { obstacles radii } & R_{1}, R_{2} & 0.2,0.4 \\ \text { particles radii } & r & 0.005(p b c) \text { or } 0.01\left(\frac{1}{2} p b c\right) \\ \text { occupied volume } & \delta & \frac{N V_{0}}{V-V_{o b s}} \\ \text { size } & k \times l & 1 \times 1(p b c) \text { or } 2 \times 2\left(\frac{1}{2} p b c, N=2\right) \text { or } 4 \times 5\left(\frac{1}{2} p b c, N=10\right) \\ \text { particles number } & N & 2 \text { or } 10\end{array}$

The following dynamical quantities are particularly interesting for the qualitative picture of the motions.

- The average timing $t_{0}$ of the collisions, equal to the future average $\langle t(\cdot)\rangle_{+}$of the time $t(x)$ elapsing between two successive collisions.

-The collision rate $\nu$ will be the number of collisions between moving particles divided by the total number of collisions including the ones with the obstacles and the walls (the latter are present only in the $\frac{1}{2} p b c$ case).

-The average entropy creation per collision, equal to the future average $t_{0}\langle\sigma\rangle_{+}$.

-The Lyapunov exponents $\lambda_{\max }$ and $\lambda_{\min }$ defined, respectively, by the largest expansion rates of line elements under the action of the positive iterates of the evolution map $S$ or by the minimum absolute value of the expansion or contraction rates.

- The entropy correlation time defined by the decay rate $\left\langle\sigma_{\tau}\left(S^{n} x\right) \sigma_{\tau}(x)\right\rangle_{+}=O\left(e^{-\vartheta n}\right)$ of the entropy autocorrelation (recall that $Q_{t}$ denotes the continuous time evolution, see $\S 1$ ).

The data below have been obtained empirically, i.e. without any attempt at estimating errors, and are useful to get an idea of the basic qualitative properties of the system. For $N=2$ particles with $e_{0}=1 / 2, m=L=1, E=1$, density, $k$, $\ell$, and radii as above: 


$\begin{array}{lccc} & & p b c, k=1 & \frac{1}{2} p b c, k=2 \\ \rho & \text { density } & 2 & 0.5 \\ \delta & \text { occup. vol. } & 4.23 \cdot 10^{-4} & 4.23 \cdot 10^{-4} \\ \lambda_{\max }^{-1} & & 1.32 & 1.31 \\ \lambda_{\min }^{-1} & & 1.53 \cdot 10^{1} & 2.16 \cdot 10^{1} \\ \vartheta^{-1} & \forall \tau & \leq 20 & \leq 20 \\ t_{0} & \text { timing pace } & 1.50 \cdot 10^{-1} & 1.35 \cdot 10^{-1} \\ \nu & \text { collision rate } & 1.09 \cdot 10^{-2} & 4.93 \cdot 10^{-3} \\ \frac{t_{0}}{2 N-1}\langle\sigma\rangle_{+} & \text {entropy prod. } & 1.75 \cdot 10^{-2} & 1.49 \cdot 10^{-2}\end{array}$

where only the errors (not shown, but amounting to less than $0.1 \%$; see below) on $t_{0},\langle\sigma\rangle_{+}$have been measured with care since we need them in our experiments. The other data are purely indicative of the orders of magnitude; $\forall \tau$ means for all values of $\tau$ considered below.

For 10 particle systems:

$\begin{array}{lccc} & & p b c, k=1 & \frac{1}{2} p b c, k=2 \\ \rho & \text { density } & 10 & 0.5 \\ \delta & \text { occup. vol. } & 2.11 \cdot 10^{-3} & 4.23 \cdot 10^{-4} \\ \lambda_{\max }^{-1} & & 3.50 & 4.71 \\ \lambda_{\min }^{-1} & & 3.12 \cdot 10^{2} & 2.20 \cdot 10^{3} \\ \vartheta^{-1} & \forall \tau & <20 & <20 \\ t_{0} & \text { timing pace } & 2.87 \cdot 10^{-2} & 2.8 \cdot 10^{-2} \\ \nu & \text { pair collisions } & 8.95 \cdot 10^{-2} & 8.92 \cdot 10^{-3} \\ \frac{t_{0}}{2 N-1}\langle\sigma\rangle_{+} & \text {entropy prod. } & 4.07 \cdot 10^{-3} & 3.52 \cdot 10^{-3}\end{array}$

with the same comments on the errors and the symbols as presented in (2.8).

We shall study the probability distribution $\pi_{\tau}(p) d p$, in the stationary state $\mu$, of the variable $p$ that is defined by (2.6) above for $\tau$ large. In fact the theory of the chaotic hypothesis foresees that, if $\tau$ is large compared to $\lambda_{\min }^{-1}$, then $\pi_{\tau}(p)$ verifies:

$$
\log \frac{\pi_{\tau}(p)}{\pi_{\tau}(-p)}=\tau t_{0}\langle\sigma\rangle_{+} p
$$

This is the content of the fluctuation theorem discussed in [GC1],[CG2],[G2],[G1] and it means that the odd part of $\log \pi_{\tau}(p)$ is linear in $p$ with an a priori determined slope. Nothing is known about the even part.

One may think that the even part is proportional to $p^{2}$, i.e. $\log \frac{\pi_{\tau}(p)}{\pi_{\tau}(1)}=-\frac{1}{4}(p-1)^{2} \tau t_{0}\langle\sigma\rangle_{+}$; therefore it is interesting to check whether the kurtosis:

$$
\kappa_{\tau}=\frac{\left\langle(p-1)^{4}\right\rangle_{+}-3\left\langle(p-1)^{2}\right\rangle_{+}^{2}}{\left\langle(p-1)^{2}\right\rangle_{+}^{2}}
$$

vanishes, if $\langle\cdot\rangle_{+}$denotes average with respect to the SRB distribution $\mu$ in (1.1) (recall that $\kappa_{\tau} \equiv 0$ for a Gaussian distribution and the value $\kappa_{\tau}$ can be taken as a quantitative dimensionless estimate of the "non gaussian" nature of the distribution). The central limit theorem for transitive Anosov systems, [S], implies a Gaussian distribution for the variable $\sigma_{\tau}$ for large $\tau$; 
this yields information about the deviations of $\tau \sigma_{\tau}$ from its average $\tau\langle\sigma\rangle_{+}$by quantities of order $\sqrt{\tau}$; but (2.10) describes properties of large deviations, proportional to $\tau$. Therefore there is no a priori reason to expect that the $\pi_{\tau}(p)$ is Gaussian; hence we do not expect that $\kappa_{\tau}=0$; and the evaluation of $\kappa_{\tau}$, once (2.10) is established, is of considerable interest.

Note that the variable $\sigma_{\tau}$ varies on a finite range, at fixed $N$. This means that $p$ varies in a finite interval $\left[-p^{*}, p^{*}\right]$, symmetric by the time reversal symmetry, whose size can be easily measured and an idea of its value can be obtained from the following rough data for $\tau=20$ :

$$
\begin{array}{lllll} 
& p b c, N=2 & \frac{1}{2} p b c, N=2 & p b c, N=10 & \frac{1}{2} p b c, N=10 \\
p^{*} & 9.91 & 9.25 & 7.92 & 8.55
\end{array}
$$

that give the values of $p^{*}$ on the actually observed trajectories. The definition of $\alpha$ and (2.5), by using the Schwartz inequality, imply a bound:

$$
p^{*} \leq \frac{2 N-1}{\langle\sigma\rangle_{+}} \frac{E}{\sqrt{2 e_{0}}} \frac{t_{\max }}{t_{0}}
$$

if $e_{0}$ is the energy per particle and $t_{\max }$ is the maximum time between collisions. And the bound is saturated when all the particles have the same velocity parallel to the field.

Finally the result (2.10) is valid in the limit $\tau \rightarrow \infty$ and this means that, in order to check it, one has to perform many experiments with various values of $\tau$ : one expects that $\tau$ should be large compared to $\vartheta^{-1}$, at least at fixed $p$ (but the errors are not expected to be uniform in $p$ so that one should not be surprised to see still corrections at large values of $p$ for values of $\tau$ for which (2.10) holds without appreciable corrections at small values of $p$ ).

In this experiment we have computed the distribution $\pi_{\tau}(p)$ at fixed $\tau$ (using the discrete evolution $S$ ) by measuring $p$ over time intervals of length $\tau$ but spaced by a fixed number of collisions $\Delta$ during which no measurement is made. The time interval $\Delta$ has been taken large compared to the relevant characteristic times of the system, i.e. the average free flight time or the inverse of the time decay constant for the entropy autocorrelation function. The latter times being of the same order of magnitude and of the order of 1 to 10 collision times, we took $\Delta=50$ collisions, see (2.8),(2.9). Larger $\Delta$ would have been better: but we would lose statistics. We then assume, in the statistical analysis, that the data so obtained are uncorrelated.

We made some empirical tests that this time delay was sufficiently large by investigating, in various cases, how important the time correlations were in the evaluation of the errors. Not unexpectedly we found that the statistical errors decrease by increasing the sampling delay $\Delta$, in spite of the smaller statistical samples: so as far as statistical errors are concerned there is some (small) advantage in taking measurements spaced by $\Delta$ large. But mainly this was a test of our independence assumption used in the errors theory. If there had been a drastic change in the statistical errors we would have concluded that the correlation time for the entropy production was not of the order of 10 collision times.

Another time scale of interest is $\lambda_{\min }^{-1}$ : this is however very difficult to measure and it may have value $+\infty$ for some values of $E$, (see Fig.15 below). There is no evidence that this time scale is related to the entropy autocorrelation: which appears to be short ranged, as far as we can see. On the other end there is evidence that as $E$ grows the number of positive Lyapunov exponents decreases. In $\S 6$ we try to establish a connection between this observation and the fluctuation theorem.

This is very important for us as it shows that the chaotic hypothesis may hold in a far stronger sense than originally meant in [GC1], [GC2]. One can see that if the dimension of the stable manifold of the attractor points is not equal to that of the unstable manifold, then the closure of attractor for the forward motion cannot be the same as that of the attractor for the backward motion. Hence the time reversal cannot leave the attractor invariant but the chaotic hypotesis, 
as formulated in $\S 1$, tells us that nevertheless the motion on the attractor is reversible in the sense that there is a map $i^{*}$ which leaves the attractor invariant and changes $S$ into $S^{-1}$. The map $i^{*}$ (whose existence is far from obvious) will be called the local time reversal on the attractor, see $\S 6,[\mathrm{BG}]$.

\section{§3. Ising model analogy.}

The fluctuation theorem as expressed by (2.10) and the subsequent comments on the Gaussian nature of the function $\pi_{\tau}(p)$ may seem somewhat strange and unfamiliar.

It is therefore worth pointing out that the phenomenon of a "linear fluctuation law" on the odd part of the distribution, in the sense of (2.10), without a globally Gaussian distribution, is in fact well known in statistical mechanics and probability theory. And an example of what the fluctuation theorem means in a concrete case, in which $\pi_{\tau}(p)$ is not Gaussian, can be made by using the Ising model on a 1 dimensional lattice $Z$.

We consider the space $\mathcal{C}$ of the spin configurations $\underline{\sigma}=\left\{\sigma_{\xi}\right\}, \xi \in Z$ and the map $S$ that translates each configuration to the right (say). The "time reversal" is the map $i:\{\underline{\sigma}\} \rightarrow\{-\underline{\sigma}\}$ that changes the sign to each spin.

The probability distribution that approximates the SRB distribution is the finite volume Gibbs distribution:

$$
\mu_{\Lambda}(\underline{\sigma})=\frac{\exp \left(J \sum_{j=-T}^{T-1} \sigma_{j} \sigma_{j+1}+h \sum_{j=-T}^{T} \sigma_{j}\right)}{\text { normalization }}
$$

where $\Lambda=[-T, T]$ is a large interval, $J, h>0$. The configuration $\underline{\sigma}$ outside $\Lambda$ is distributed independently on the one inside the box $\Lambda$, to fix the ideas.

Calling $\langle m\rangle_{+}$the average magnetization in the thermodynamic limit we define the magnetization in a box $\left[-\frac{\tau}{2}, \frac{\tau}{2}\right]$ to be $M_{\tau}=\tau\langle m\rangle_{+} p$ and we look at the probability distribution $\pi_{\tau}^{T}(p)$ of $p$ in the limit $T \rightarrow \infty$. The Gibbs distribution corresponding to the limit of (3.1) will play the role of the SRB distribution. Calling this limit probability $\pi_{\tau}(p)$ it is easy to see that:

$$
\frac{\pi_{\tau}(p)}{\pi_{\tau}(-p)} \underset{\tau \rightarrow \infty}{\longrightarrow} e^{2 \tau h\langle m\rangle+p}
$$

This is in fact obvious if we take the two limits $T \rightarrow \infty$ and $\tau \rightarrow \infty$ simultaneously by setting $T=\frac{\tau}{2}$. In such a case, if $\sum_{\underline{\sigma} ; p}$ denotes summation over all the configurations with given magnetization in $[-T, T]$, i.e. such that $\sum_{j=-\frac{\tau}{2}}^{\frac{\tau}{2}-1} \sigma_{j}=\langle m\rangle_{+} p$ the distribution (3.1) gives us immediately that:

$$
\frac{\pi_{\tau}^{T}(p)}{\pi_{\tau}^{T}(-p)}=\frac{\sum_{\underline{\sigma}, p} \exp J \sum_{j=-T}^{T-1} \sigma_{j} \sigma_{j+1}+h \sum_{j=-T}^{T} \sigma_{j}}{\sum_{\underline{\sigma},-p} \exp J \sum_{j=-T}^{T-1} \sigma_{j} \sigma_{j+1}+h \sum_{j=-T}^{T} \sigma_{j}} \equiv e^{2 \tau h\langle m\rangle_{+} p}
$$

if we use the symmetry of the pair interaction part of the energy under the "time reversal" (i.e. under spin reversal).

The error involved, in the above argument, in taking $T=\frac{\tau}{2}$ rather then first $T \rightarrow \infty$ and then $\tau \rightarrow \infty$, can be easily corrected since the corrections are "boundary terms", and in one dimensional short range spin systems there are no phase transitions and the boundary terms have no influence in the infinite volume limit (i.e. they manifest themselves as corrections that vanish, as $T \rightarrow \infty$ followed by $\tau \rightarrow \infty$ ).

One may not like that the operation $i$ commutes with $S$ rather than transforming it into $S^{-1}$. Another example in which the operation $i$ does also invert the sign of time is obtained 
by defining $i$ as $\left.\{i \underline{\sigma}\}\right|_{j}=-\sigma_{-j}$ : the (3.3) can be derived also by using this new symmetry operation.

The above examples show why there is a priori independence between any Gaussian property of $\pi_{\tau}(p)$ and the fluctuation theorem. The theory of the fluctuation theorem in [GC1] is in fact based on the possibility (discovered in $[\mathrm{S}]$ ) of representing a chaotic system as a one dimensional short range system of interacting spins (in general higher that $\frac{1}{2}$ ); and the argument is, actually, very close to the above one for the Ising model with, however, a rather different time reversal operation. See [G2] for mathematical details on the boundary condition question. 


\section{§4. Experimental results.}

We have made several measurements. Each measurement is quite delicate and time consuming, hence the reader will probably forgive us for not having done all the experiments that one finds natural to do. Each experiment requires several days of CPU time on the computers that we used (and several months to prepare the final runs). The statistical errors have been measured by 3 times the standard deviation, and the other errors are estimated by following the criteria discussed in $[\mathrm{GG}]$ and resumed in the appendix. Thus the experiments should be reproducible within our error bars if the latter are defined as we do. Hopefully the data we give can be of use even if one decides (for mathematical reasons or to test other theoretical ideas) to change the assignments of the errors. In all the following graphs the error bounds are always marked although sometimes they may be not visible.

4.1. Periodic boundary conditions, $N=2$ : The values of $R_{1}, R_{2}$ are respectively $0.2,0.4$; the particle radius is 0.005 and the electric field is fixed $E=1$., a value that seems to be quite large (see however §6). The qualitative data of the resulting evolution are given in the first column of the table (2.8). Other experiments at varying field intensity are described in $\S 6$.

4.1.1 The probability distribution $\pi_{\tau}(p)$ : The evolution is studied over $1.08 \cdot 10^{6}$ collisions. In Fig.2 we give the graph of $\pi_{\tau}(p)$ for various $\tau$.
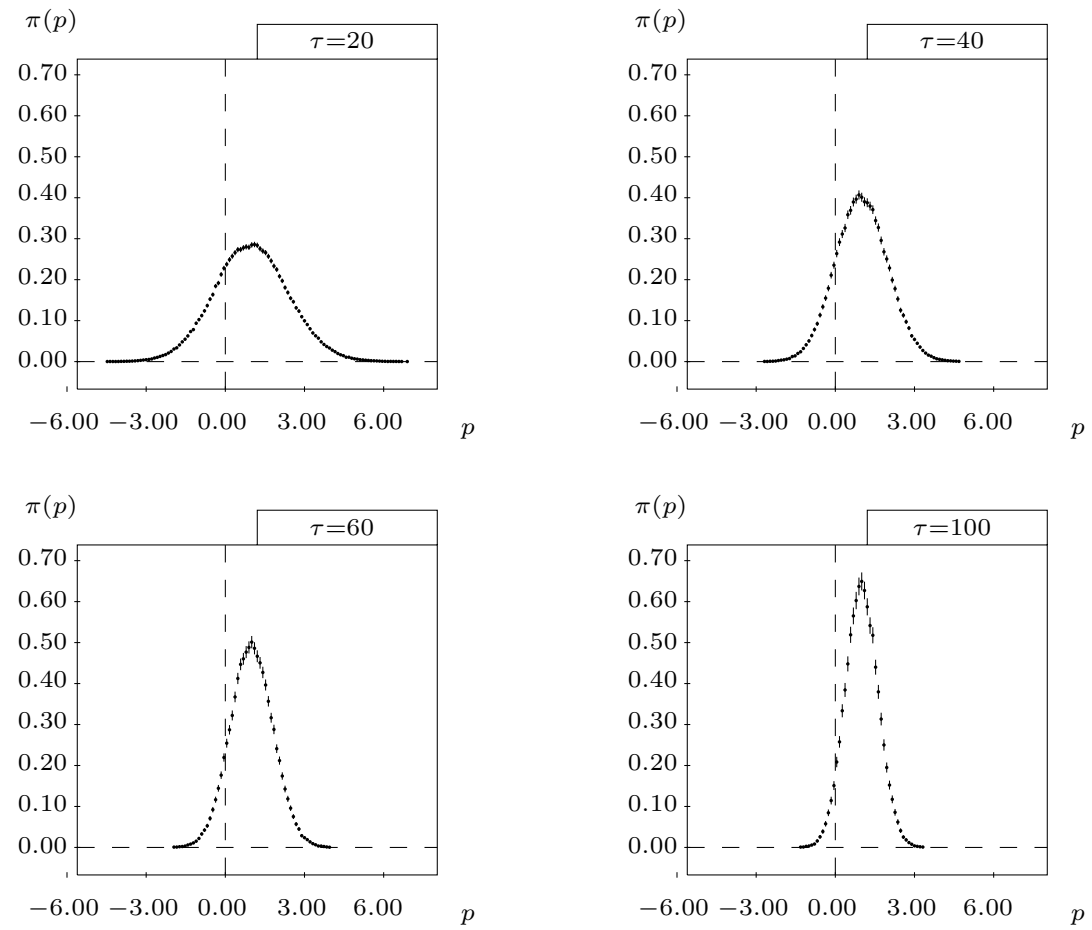

Fig.2: The histograms for $\pi_{\tau}(p)$ at various values of the observation time $\tau=20,40,60,100$. Each vertical bar is the error bar centered around the measured point. The dots on the axis mark the extremes of the interval where the observed data differ form 0 within the statistical error.

The error bars are very small particularly for the data at the edge of the observability interval because the data are very many. But the relative errors (not shown) are very small at the center and they are very large at the edges, of course. 
We have attributed to each point on the above histograms a statistical error as explained in $\S 5$ below (essentially they have been supposed independent variables and they have been given an error of 3 times the standard deviation). In fact the analysis of dispersion of each value shows that the "law of large numbers" is obeyed, and the standard deviation $m_{n}(\tau) \stackrel{\text { def }}{=}\left\langle\left(p-\langle p\rangle_{\tau}\right)^{n}\right\rangle$, $n=2$, and the third order deviation, $n=3$, approach 0 with an apparent decay given by:

$$
\begin{aligned}
& m_{2}(\tau)=-.005( \pm .002)+39.80( \pm 0.05) \frac{1}{\tau} \\
& m_{3}(\tau)=-.002( \pm .004)+93 .( \pm 1.5) \frac{1}{\tau^{2}}
\end{aligned}
$$

The error analysis leading to (4.2) follows the same scheme of [GG]. We can also use here the notion of "goodness" introduced in [GG] to measure how good a fit is (reproduced in the appendix below), and we measured the goodness of the above fits. We do not use the word "reliability test", and use "goodness" instead, to avoid inducing the reader to think that we rely on some standard error analysis. The goodness is $2.45 \cdot 10^{-3}$ and $4.94 \cdot 10^{-3}$ respectively. The results are given in the following Fig.3:
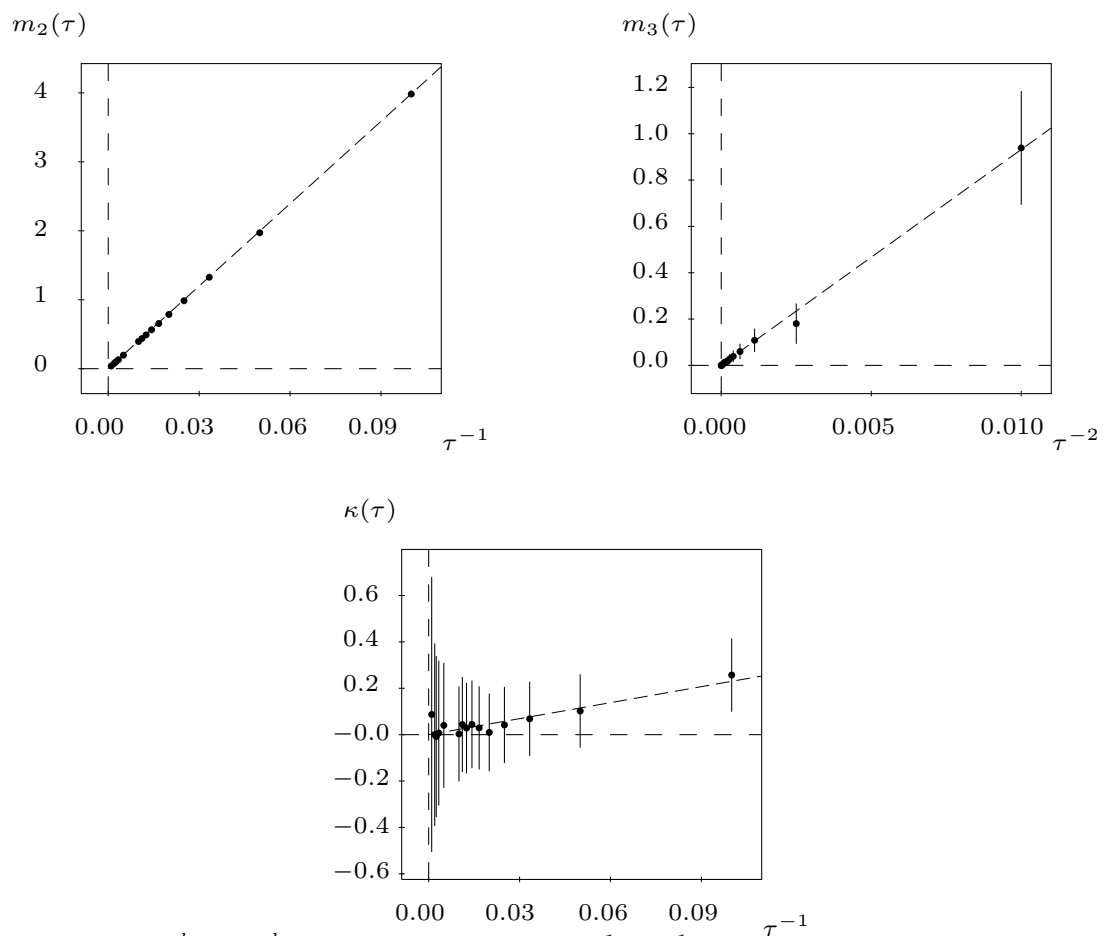

Fig.3: The decay of the $2^{d}$ and $3^{d}$ moments, as functions of $\frac{1}{\tau}$ or $\frac{1}{\tau^{2}}$, and the kurtosis.

The question of whether the probability distribution $\pi_{\tau}(p)$ is Gaussian is investigated by computing the kurtosis as a function of $\tau$ : the latter quantity is a dimensionless parameter and it is related to the fourth moment: $\frac{\left\langle\sigma_{\tau}^{4}\right\rangle-3\left\langle\sigma_{\tau}^{2}\right\rangle^{2}}{m_{2}(\tau)}$. It can be fitted by a law:

$$
\kappa(\tau)=\left[0.00( \pm 0.01)+2.3( \pm 0.3) \frac{1}{\tau}\right] \times \frac{1}{m_{2}(\tau)^{2}}
$$


The data are reported in Fig.3. But, unlike the cases of $m_{2}(\tau)$ and $m_{3}(\tau)$ the goodness of this fit is $1.63 \cdot 10^{-2}$ and it is comparable with the goodness of fits with a law $\frac{1}{\tau^{2}}$ or even $\frac{1}{\tau^{3}}$ : the errors are too big so that many fits are "as good" (and also very good: this only shows that the notion of goodness has shortcomings as much as any other accuracy test).

We conclude that the distribution seems compatible with a Gaussian. Of course one expects a Gaussian behaviour for the small deviations, i.e. for $(p-1) \sim \tau^{-1 / 2}$ : If one assumes that the system is Anosov (or just that it has an Axiom A attractor) then this follows, as a theorem, from the results of [S] (or [R2]).

However we know that it cannot be Gaussian beyond the range $O\left(\tau^{-1 / 2}\right)$, because it has support between $\pm p^{*}$ with $p^{*}<+\infty$, see (2.12) and $\S 3$. Hence we the apparent closeness to a Gaussian distribution might be an accident, that might disappear as $\tau$ increases. If it does not then this is an interesting question to examine, see $\S 6$.

In Fig.4 we show the main quantity of interest here, i.e. the graph of:

$$
x(p)=\frac{1}{\tau t_{0}\langle\sigma\rangle_{+}} \log \frac{\pi_{\tau}(p)}{\pi_{\tau}(-p)}
$$

versus $p$ (recall that $\langle\sigma\rangle_{+}$is $(2 N-1) j$ if $j$ is the electric current):
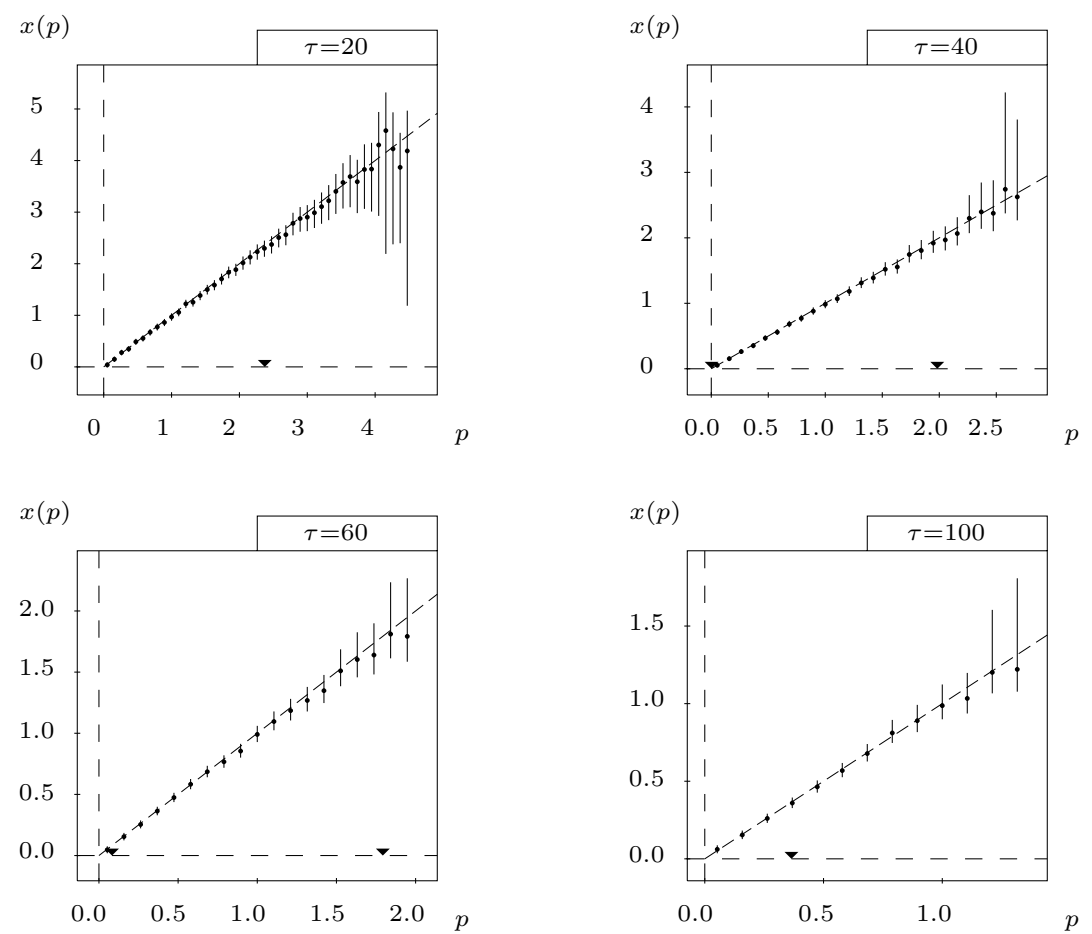

Fig.4: The fluctuation theorem test for $\tau=20,40,60,100$. The dashed straight line is the theoretical prediction of the fluctuation theorem. The arrows mark the point at distance $\sqrt{\left\langle(p-1)^{2}\right\rangle}$ from 1. The error bars are inherited from the histogram data in Fig.2.

The abscissae axis is discretized and the number of events in which $p$ falls in a given interval is taken as proportional to $\pi_{\tau}(p)$. Therefore Fig.4 is a histogram.

All values corresponding to different $\tau$ collapse on a single straight line in the interval $p \in[0,1.5]$ in the worst cases (i.e. largest $\tau$ ). For higher values of $p$ (depending on $\tau$ ) the statistics becomes gradually more and more poor as the deviations from the mean value of $p$ become too large. 
4.1.2. Conclusions: From the above data we infer that one cannot exclude that the distribution is Gaussian. Assuming that it is Gaussian then the fluctuation theorem predicts a standard deviation $m_{2}(\tau)=\frac{2}{\tau t_{0}\langle\sigma\rangle_{+}} \equiv \frac{A}{\tau}$, see the lines preceding (2.11) and the above experimental data give:

$$
\begin{array}{ll}
A=37.96 \pm .21 & \text { (Gaussian assumption) } \\
A=39.80 \pm .05 & \text { (experiment) }
\end{array}
$$

where the Gaussian value is computed from the experimentally measured $\langle\sigma\rangle_{+}$and $t_{0}$ (to which we attribute a statistical error of 3 times the standard deviation), while the experimental value is computed from $m_{2}(\tau)$, see (4.2); the error on the second line is as implied by (4.2).

The standard deviations of $p$ from the value 1 are for $\tau=20,40,60,80,100$ respectively 1.41, $0.99,0.81,0.70$ and 0.63 . Such values, multiplied by 3 (recall that our conventional statistical errors are 3 times the standard deviation), can be arbitrarily assumed to be the boundary between the small and the large deviations.

Note that, as already mentioned in $\S 2$, if the chaotic hypothesis is assumed then we know from the theory of Sinai that the small deviations obey a Gaussian law as $\tau \rightarrow \infty$ : but the theory in general does not predict the standard deviation. The example of $\S 3$ is a good illustration, we believe, of the situation: in that case too we have a good understanding of the odd part of the distribution, but no grip on the even part and no reason to know a priori the distribution of the magnetization (i.e. also its even part). The results may mean that the values of $\tau$ that we reach are not large enough to test the validity of the central limit theorem. On the other hand the fluctuation theorem that follows from the chaotic hypothesis is independent on the central limit theorem (as the example in $\S 3$ suggests and illustrates) and therefore the above results are, in our opinion, a good test of the chaotic assumption. We shall examine this point in more detail in $\S 6$.

Of course the choice, mentioned above, of 3 standard deviations to measure the statistical errors and the large deviations "threshold" is arbitrary. One could, as very often done, decide to use 1 standard deviation instead. Then we could say that we can go quite far in the large deviation region, but on the other hand many error bars become too small to be compatible with the data. This is a well known problem with all experiments and we can just report that it appears also in the present experiment. It could only be solved by better experiments: hence we decided to perform experiments in which the computing facilities available to us were pushed further. The results will be described below (when discussing the case of semi-periodic boundary conditions and $N=2,10)$.

$\S 4.2$ Periodic boundary conditions, $N=10$ : The geometry is the same as in the previous case. The values of $R_{1}, R_{2}$ are respectively $0.2,0.4$; the particle radius is 0.005 and the electric field is fixed $E=1$.. The qualitative data of the resulting evolution are given in the first column of the table (2.9).

4.2.1 The probability distribution $\pi_{\tau}(p)$ : The evolution is studied over $7.57 \cdot 10^{7}$ collisions. In Fig.5 we give the graph of $\pi_{\tau}(p)$ for various $\tau$. 

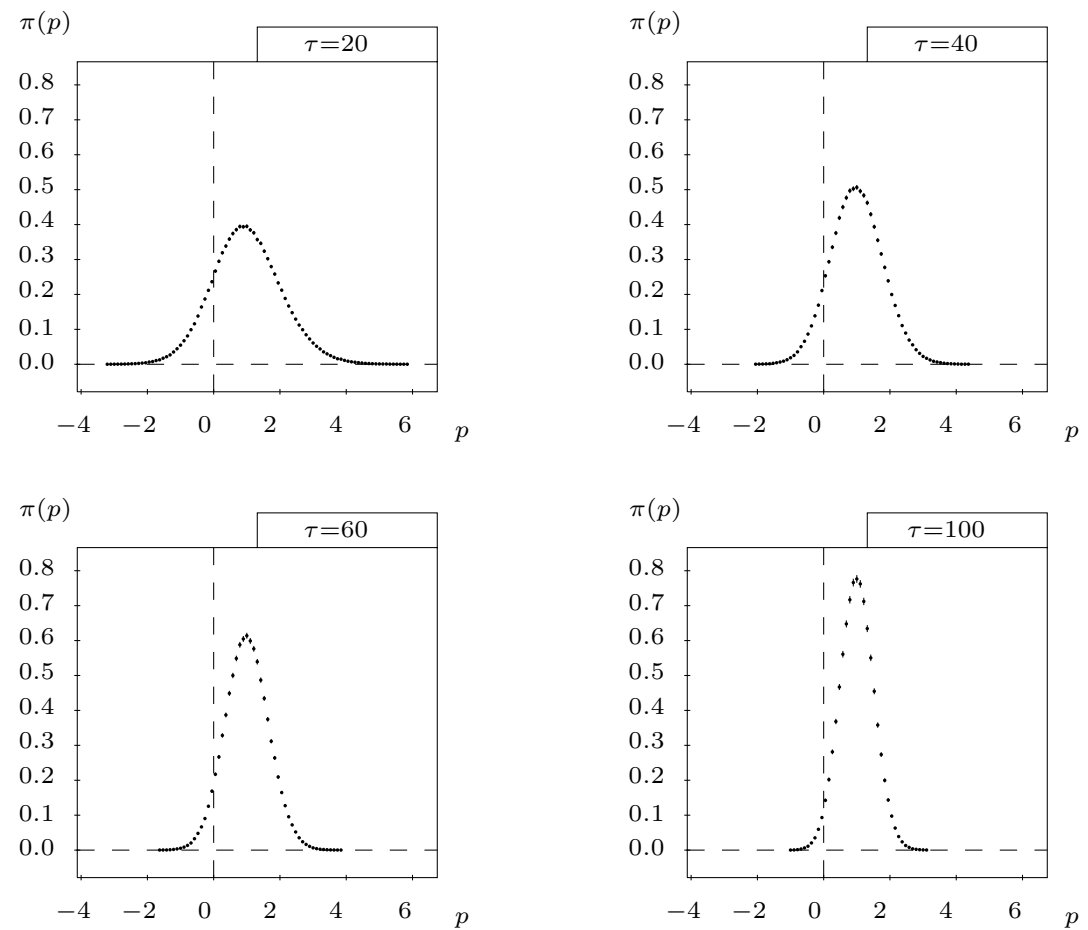

Fig.5: The distribution $\pi_{\tau}(p)$ for $\tau=20,40,60,100$.

and in Fig.6 we give the standard deviation and the third order deviation, as in the previous case as functions of $\tau^{-1}$ and $\tau^{-2}$ respectively.
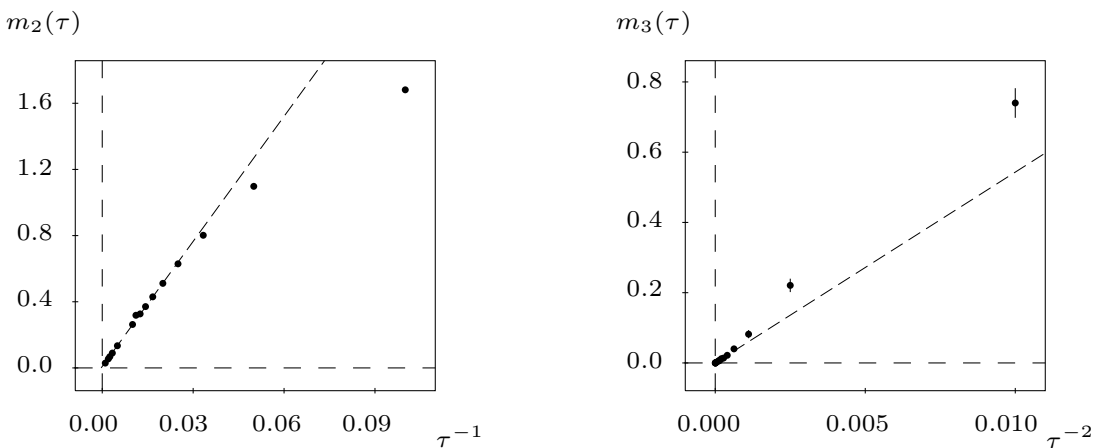

$\kappa(\tau)$

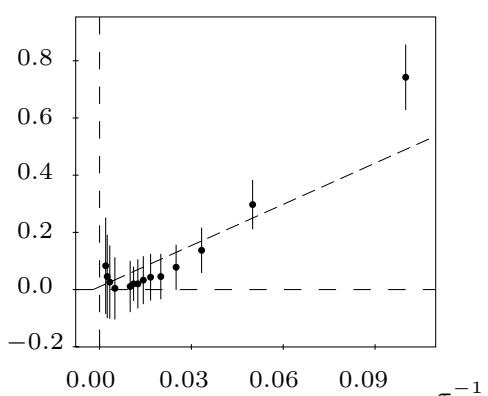

Fig.6: The decay of the $2^{d}$ and $3^{d}$ moments, as functions of $\frac{1}{\tau}$ or $\frac{1}{\tau^{2}}$ and $\tau^{-1}$ the kurtosis as a function of $\frac{1}{\tau}$. 
The fits give:

$$
\begin{aligned}
& m_{2}(\tau)=0.009( \pm .005)+25.24( \pm 0.4) \frac{1}{\tau} \\
& m_{3}(\tau)=-.0002( \pm .0003)+54.3( \pm 1.5) \frac{1}{\tau^{2}}
\end{aligned}
$$

and the goodness of the above fits is $1.2 \cdot 10^{-3}$ and $3.4 \cdot 10^{-4}$ respectively, for the data with $\tau>25$. The data with $\tau<25$ deviate from the above law and we interpret this as finite size effects (expected from the theory but absent in the previous case already for $\tau>25$ ).

In Fig. 6 the kurtosis graph is reported: for which we attempted a best fit as:

$$
\kappa(\tau)=-0.01( \pm 0.02)-4.8( \pm 0.9) \frac{1}{\tau}
$$

with a goodness of $4.03 \cdot 10^{-4}$. The data are not many because the experiment is hard (in terms of CPU time).

Finally the main quantity of interest, i.e. the graph of $x(p)=\frac{1}{\tau t_{0}\langle\sigma\rangle_{+}} \log \frac{\pi_{\tau}(p)}{\pi_{\tau}(-p)}$ versus $p$ :
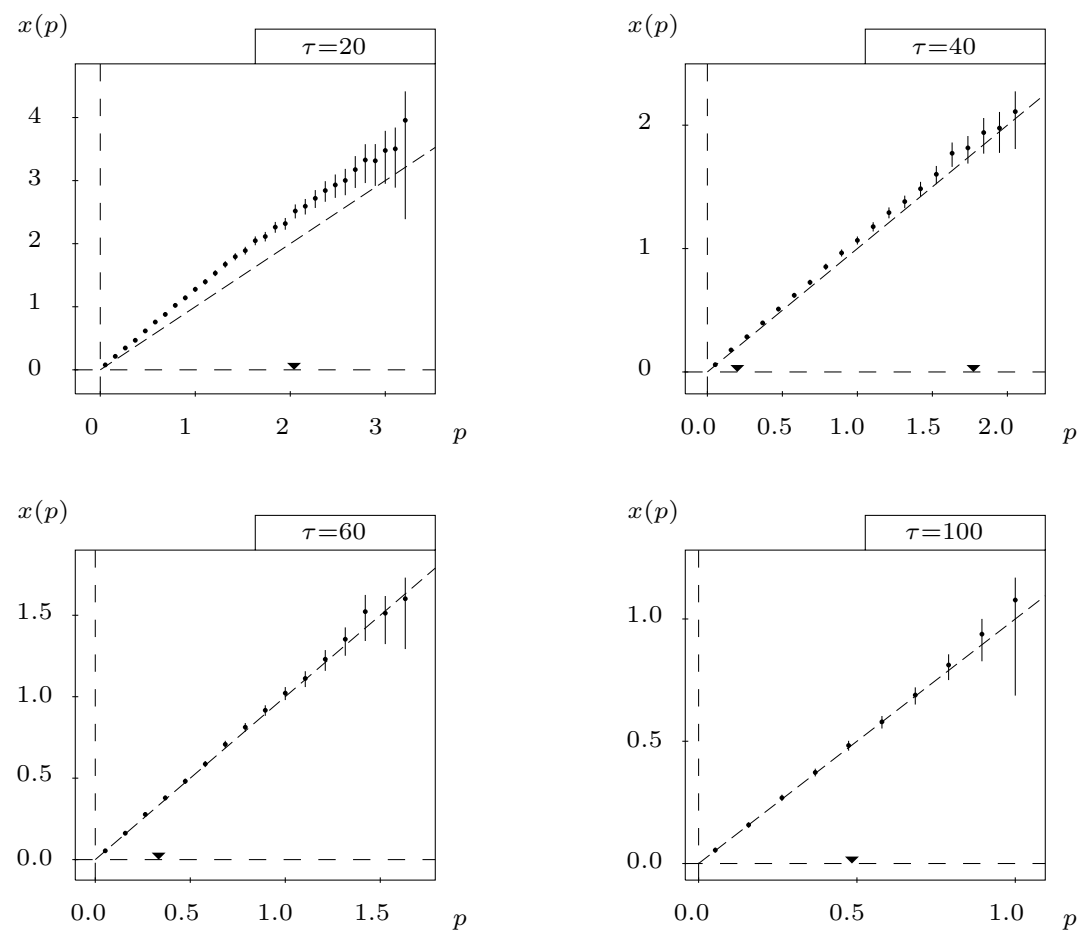

Fig.7: The graphs for the fluctuation theorem test for $\tau=20,40,60,100$. The dashed straight line is the theoretical prediction of the fluctuation theorem. The arrows mark the point at distance $\sqrt{\left\langle(p-1)^{2}\right\rangle}$ from 1 . The error bars are inherited from the histogram data in Fig.5.

The abscissae axis is discretized and the number of events in which $p$ falls in a given interval is taken as proportional to $\pi_{\tau}(p)$. Therefore Fig. 7 is a histogram.

It is remarkable that the finite size effects, i.e. the manifestation of important deviations from the linear law for "small" $\tau$ (as we interpret them), are here very clear: the case $\tau=20$ does not follow the scaling, in contrast to $\tau=40,60,80,100,(\tau=80$ is not shown). 
\$4.1.4 Conclusions, $N=10$ : The case $N=10$ is very similar to the case $N=2$ as the theory predicts. From the above data we cannot exclude that the distribution is Gaussian in the observed range of values of $p$. Assuming that it is Gaussian then the theory gives a standard deviation $m_{2}(\tau)=\frac{2}{\tau t_{0}\langle\sigma\rangle_{+}} \equiv \frac{A}{\tau}$, see (2.11) and the above experimental data give:

$$
\begin{aligned}
& A=25.82 \pm .02 \quad \text { (Gaussian assumption) } \\
& A=25.24 \pm .4 \quad \text { (experiment) }
\end{aligned}
$$

where the theoretical value is computed from the experimentally measured $\langle\sigma\rangle_{+}, t_{0}$, while the experiment value is computed from the graphs for $m_{2}(\tau)$. The error analysis is carried along the same lines as that of the case $N=2$ above.

The standard deviations of $p$ from the value 1 are for $\tau=20,40,60,80,100$ respectively $\sqrt{\frac{A}{\tau}}=1.05,0.79,0.66,0.57$ and 0.51 . Such values, multiplied by 3 , can be arbitrarily assumed to be the boundary between the small and the large deviations.

The same comments to the case $N=2$ can be made here and therefore the data, in our opinion, yield a good test of the chaotic assumption in the case $N=10$ too.

$\S 4.3 .1$ Semi-periodic boundary conditions, $N=2$ : The results are very similar in the case of semi-periodic boundary conditions and the following two graphs give the experimental values over $10^{8}$ collisions (with the walls, other particles or obstacles) for the probability distribution $\pi_{\tau}(p)$ with $\tau=20,100$; the $\pi_{\tau}(p)$ at intermediate values of $\tau$ have also been measured $(\tau=$ $40,60,80)$ but we do not give the graphs.
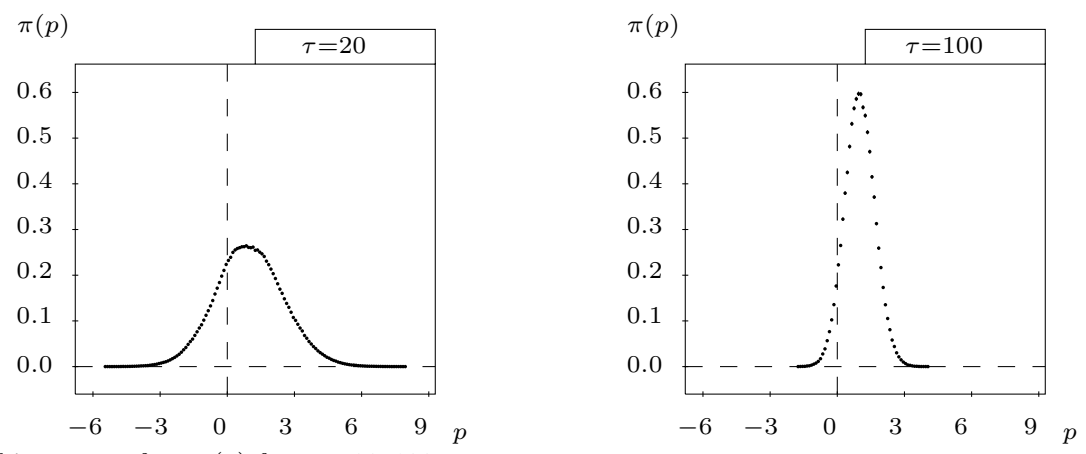

Fig.8: The histograms for $\pi_{\tau}(p)$ for $\tau=20,100$.

The errors are treated, naturally, as in the corresponding previous cases.

The next two figures give the graphs of the $x(p)$ in (4.5), which is expected to be the graph of $x(p)=p$. Again we give only the two extreme cases, $\tau=20,100$ we have been able to measure: the agreement with the "theory" is as excellent in the intermediate cases $(\tau=40,60,80)$. The errors are slightly smaller than in the previous experiments because of the difference in the length of the computed trajectory. 

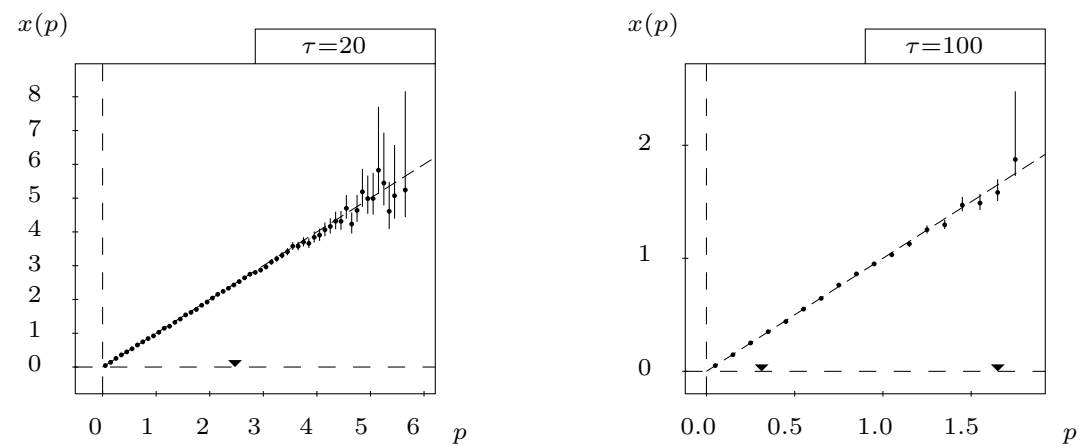

Fig.9: The graphs for the fluctuation theorem test, case of $\frac{1}{2}(p b c), N=2$; the dashed line is the fluctuation theorem prediction, $\tau=20,100$. The arrows mark the point at distance $\sqrt{\left\langle(p-1)^{2}\right\rangle}$ from 1 .

We have also measured the kurtosis and the decay of the second and third moments: the results are very similar to the ones in the periodic boundary conditions case and can be commented in the same way.
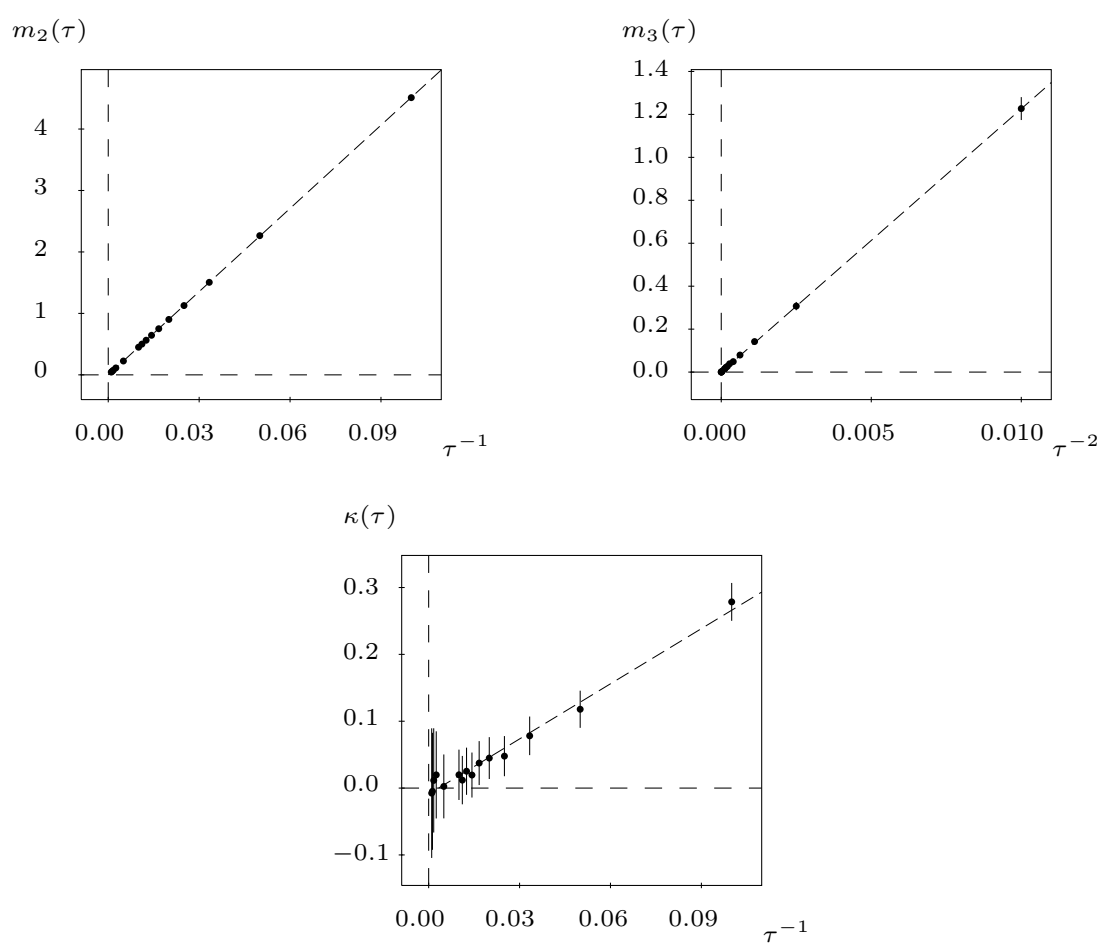

Fig.10: The second moment, the third moment and the kurtosis.

The fits are:

$$
\begin{aligned}
& m_{2}(\tau)=-0.00( \pm 0.002)+45.1( \pm 0.01) \frac{1}{\tau} \\
& m_{3}(\tau)=0.001( \pm 0.004)+122.6( \pm 10.4) \frac{1}{\tau^{2}} \\
& \kappa(\tau)=-0.009( \pm 0.04)+2.7( \pm 0.8) \frac{1}{\tau}
\end{aligned}
$$

and the goodness (see above, and the Appendix, for the definition) are $3 \cdot 10^{-3}, 2 \cdot 10^{-3}$ and $1 \cdot 10^{-2}$, respectively. 
As in the previous cases we can assume that is a Gaussian and we see that the results are:

$$
\begin{array}{ll}
A=43.8 \pm 0.03 & \text { Gaussian assumption } \\
A=45.1 \pm 0.01 & \text { experiment }
\end{array}
$$

As in the case of periodic boundary conditions the distribution is quite close to a Gaussian, although it cannot be such, strictly speaking (for the reasons already discussed).

\$4.3.4 Semi-periodic boundary conditions and 10 particles: We have also considered a further $N=10$ particles system, but at a density quite different from the previous one.

As in the previous case we need to take $\tau$ rather large to see the fluctuation law $x(p)=p$, see (4.5), to hold. But it turns out that although at $\tau=20$ "finite size effects" are still visible and very strong, already at $\tau=40$ they become essentially not visible.

Therefore we give the histogram for $\pi_{\tau}(p)$ at $\tau=20,40,60,100$ :
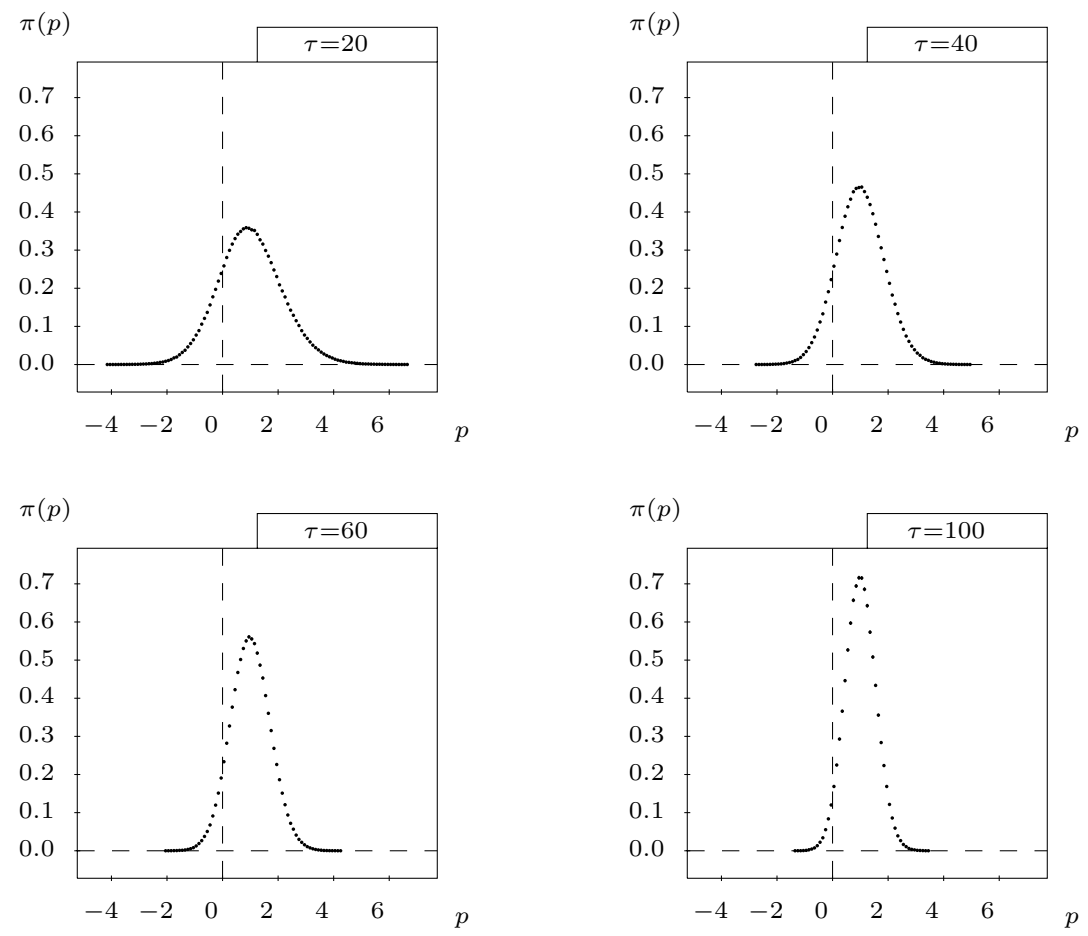

Fig.11: Histograms for $\pi_{\tau}(p)$ at $\tau=20$ and $\tau=100$. 
and the graphs of $x(p)$ at $\tau=20,40,80,100$ :
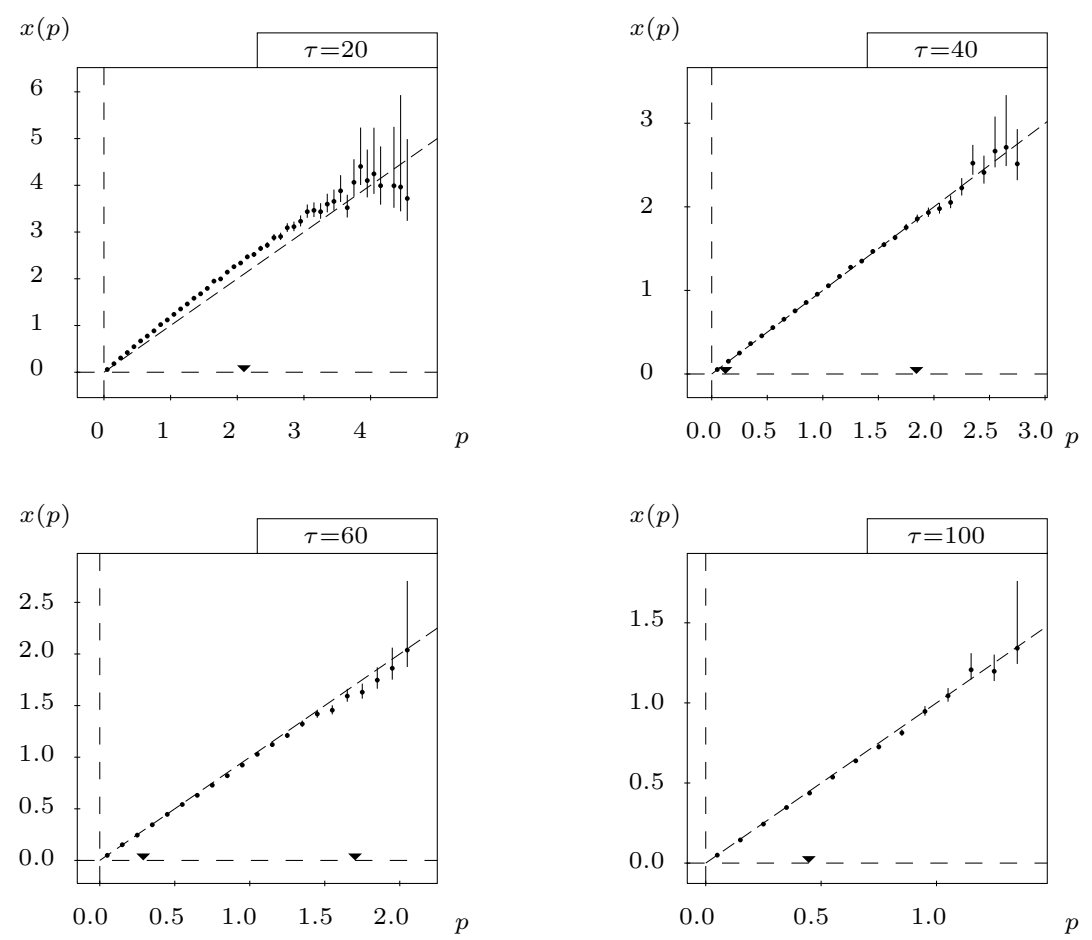

Fig.12: The linear fluctuation test, $\tau=20,40,80,100$. The dashed line is the fluctuation theorem prediction for $\tau=+\infty$. The arrows mark the point at distance $\sqrt{\left\langle(p-1)^{2}\right\rangle}$ from 1 .

which illustrate well the fact that there is a visible finite size effect that very soon becomes not observable.

The experiments were carried out over $10^{8}$ collisions. The following graphs give the kurtosis and the decay of the second and third moments. 

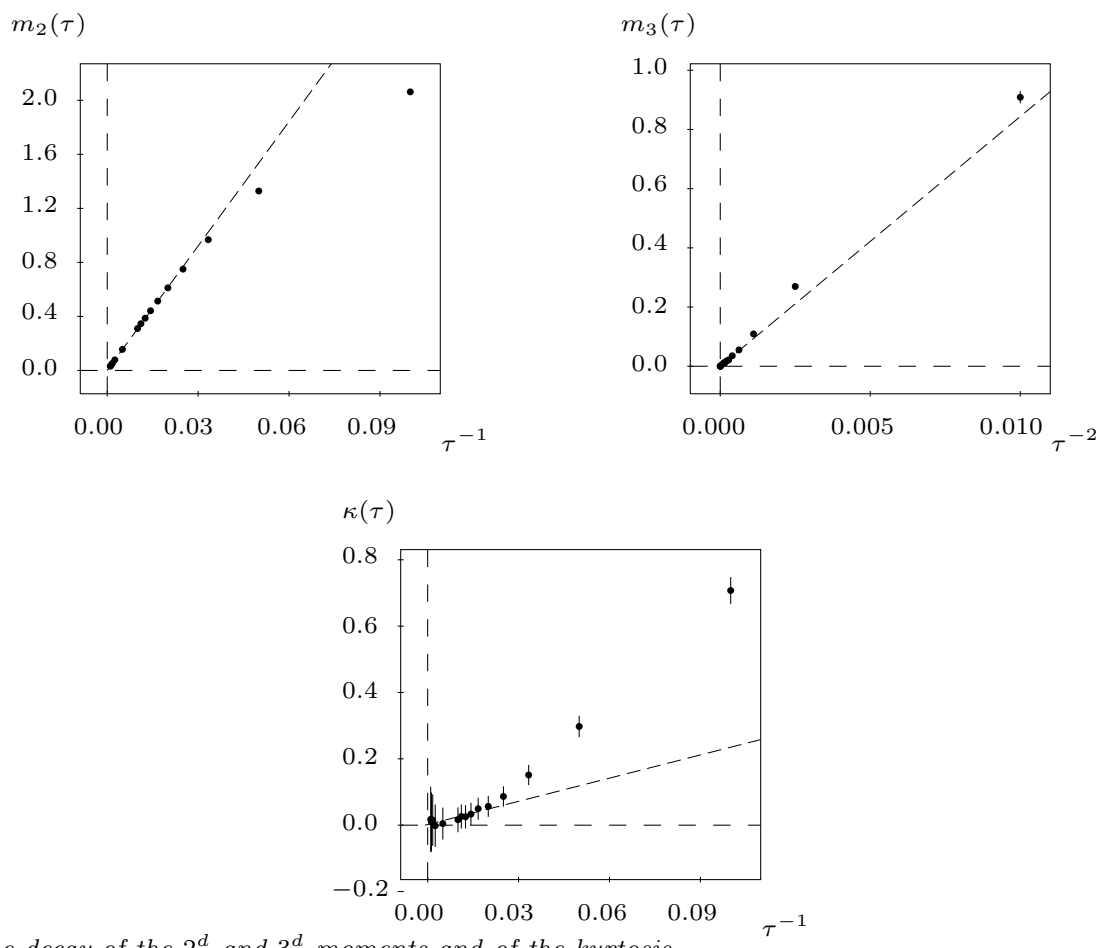

Fig.13: The decay of the $2^{d}$ and $3^{d}$ moments and of the kurtosis.

The corresponding fits are, excluding the last point that is clearly a finite size effect:

$$
\begin{aligned}
& m_{2}(\tau)=-0.002( \pm 0.0005)+30.7( \pm 0.1) \frac{1}{\tau} \\
& m_{3}(\tau)=0.0001( \pm 0.001)+84.3( \pm 11.3) \frac{1}{\tau^{2}} \\
& \kappa(\tau)=-0.00( \pm 0.07)+2.3( \pm 5.7) \frac{1}{\tau}
\end{aligned}
$$

and the goodness are $2 \cdot 10^{-3}, 1 \cdot 10^{-3}$ and $1 \cdot 10^{-2}$, respectively.

As in the previous cases if we assume that $\pi_{\tau}(p)$ is a Gaussian we see that the results are:

$$
\begin{aligned}
& A=29.2 \pm 0.002 \quad \text { (Gaussian assumption) } \\
& A=30.7 \pm 0.1 \quad \text { (experiment) }
\end{aligned}
$$

§5. Error analysis of the distribution $\pi_{\tau}(p)$.

A brief description of the methods that we use to define the errors follows. For a given $\tau$, we build a time sequence of different $p$ values:

$$
p_{n} \equiv p\left(x_{n t^{\prime}}\right)=\frac{\sigma_{\tau}\left(x_{n t^{\prime}}\right)}{\langle\sigma\rangle_{+}^{M}} \quad n=1, \ldots . M
$$

where $x_{n t^{\prime}}=S^{n t^{\prime}} x, t^{\prime}=\tau / 2+\Delta$ with $x$ randomly chosen in phase space with absolutely continuous distribution.and we have chosen $\Delta=50$ in order to decorrelate contiguous evolution data points. The $\langle\sigma\rangle_{+}^{M}$ is fixed by normalization, so that the property $M^{-1} \sum_{n=1}^{M} p\left(x_{n t^{\prime}}\right)=1$ holds, see (2.7). 
That $\Delta=50$ is a sufficient delay is warranted by the size of the entropy autocorrelation decay rate (denoted $\vartheta$ in the tables of $\S 2$ ). In general we would expect that $\Delta$ should be large compared to $\vartheta^{-1}$. An accurate determination of $\vartheta$ (including a verification of the validity of an exponential law of decay, which is not a priori obvious: see the similar problems arising for the one particle case in [GG] ) is a major enterprise and we have only made a few empirical tests on the order of magnitude of $\vartheta$ : and our choice $\Delta=50$ was dictated by purely numerical reasons as a reasonable compromise between small statistics, accuracy and computer availability. It is justified, in all our experiments, only by the empirically determined independence of the results (apart from the size of the errors) in the cases $\Delta=20$ and $\Delta=50$. We reported only the $\Delta=50$ results.

We define the discrete probability distribution:

$$
\pi_{\tau}^{D}(l ; M)=\frac{\sum_{p_{n} \in I(l)} 1}{M} \quad l=-100, . ., 100
$$

where $I(l)=[l r,(l+1) r]$ and $r=1 / 10$. The relation between the discrete and the continuous distribution when $M \rightarrow \infty$ is:

$$
\pi_{\tau}^{D}(l ; \infty)=\int_{I(l)} d p \pi_{\tau}(p)
$$

Assuming that $r$ is small enough we can expand the right hand side of (5.3):

$$
r^{-1} \pi_{\tau}^{D}(l ; \infty)=\pi_{\tau}(l r+r / 2)+\left.\frac{r^{2}}{24} \frac{\partial^{2} \pi_{\tau}(p)}{\partial p^{2}}\right|_{p=l r+r / 2}+O\left(r^{4}\right)
$$

We have checked that this $O\left(r^{2}\right)$ correction is in all our cases negligible compared to other sources of error: this has been done by approximating in (5.3) the function $\pi_{\tau}(p)$ by a Gaussian distribution (which, a posteriori, is a good approximation to $\pi_{\tau}(p)$ in the range of $p$ 's that we study):

$$
\pi_{\tau}(p) \simeq \frac{1}{\sqrt{2 \pi} \sigma} \exp \left\{-\frac{(p-1)^{2}}{2 \sigma^{2}}\right\}
$$

where $\sigma \equiv m_{2}$ is the experimental standard deviation of the distribution. By substituting (5.5) into the term with the second derivative in (5.4) we get:

$$
\pi_{\tau}(p)=r^{-1} \pi_{\tau}^{D}(l ; \infty)\left\{1-\frac{r^{2}}{24 \sigma^{2}}\left[\frac{(p-1)^{2}}{\sigma^{2}}-1\right]+O\left(r^{4}\right)\right\}
$$

with $p=l r+r / 2$.

We also have to estimate the error involved in approximating $\pi_{\tau}^{D}(l ; \infty)$ by $\pi_{\tau}^{D}(l ; M)$. Let us define

$$
P_{\tau, l}(m ; M)=\left(\begin{array}{c}
M \\
m
\end{array}\right) \pi_{\tau}^{D}(l ; \infty)^{m}\left(1-\pi_{\tau}^{D}(l ; \infty)\right)^{M-m}
$$

where $P_{\tau, l}(m ; M)$ is the probability that in $M$ elements of a sequence there exists $m$ in the interval defined by $l$. This means that we regard the various measurements of $m$ as independent: because the empirical autocorrelation of the the entropy production decays on scales smaller than $\Delta$, the interval between successive measurements.

Then, the average value of $m$ and its mean square displacement are given by:

$$
\langle m\rangle_{\tau, l}=M \pi_{\tau}^{D}(l ; \infty)
$$




$$
\left\langle\left(m-\langle m\rangle_{\tau, l}\right)^{2}\right\rangle=\frac{\pi_{\tau}^{D}(l ; \infty)\left[1-\pi_{\tau}^{D}(l ; \infty)\right]}{M}
$$

When $M$ is large enough, we shall assume that:

$$
\pi_{\tau}^{D}(l ; \infty)=\pi_{\tau}^{D}(l ; M) \pm 3\left[\frac{\pi_{\tau}^{D}(l ; M)\left(1-\pi_{\tau}^{D}(l ; M)\right)}{M}\right]^{1 / 2}
$$

is an appropriate measurement of the error. Equations (5.6) and (5.9) are the relations used in the distribution analysis.

The error analysis of the fits for $m_{2}, m_{3}, \kappa$ is the same as the one used in [GG] and it is repeated, with some minor changes to adapt it to the present sistuations, in the Appendix below.

\section{§6. Conclusions. Outlook.}

(i) Consistency and CODES: The above experiments, see Fig. 4,6,9,12 seem in "good" agreement with the theoretical predictions. We have attempted at a very accurate test compatibly with our computer resources and the need of a natural time cut-off on the duration of the experiments. The only real limitation was the computer time available; not so much as available to us but to present day technology. By using the largest existing computers our results do not seem to be substantially improvable: the motion being chaotic there is not much that one really can do without really new ideas.

Our attitude is that the theory is general and it should apply to any system like the ones described in (2.2). So in particular to our computer programs that we can refer to as the CODES. For such dynamical systems our experiments are, by definition exact and the systems are also by construction "close" to (2.2).

Therefore on such grounds we could say that we can think that the only errors in our theory are the statistical errors, i.e. our experiment is as "perfect" as one could wish.

Nevertheless, not surprisingly, the situation is more subtle. The reason is mainly that we have been unable to write CODES which "solve" (2.2) (in some sense, that is not very relevant for us here) and which at at the same time verify the time reversibility property.

We think that this is a serious flaw: because the theory, see [GC2], rests on time reversal. Strictly speaking, then, we should apply the first part of the chaotic hypothesis and only assume that the attractor verifies the Axiom A.

However for this we have no theory: the only argument that one could give, and which we do not find convincing, is that the CODES are certainly "close" approximations to (2.2). And we are used to think that close systems behave closely. The abundance of counterexamples has not deterred people to have the feeling that there is some truth to such belief (natura non facit saltus).

But since it might be simply impossible to write reversible, energy preserving, CODES what we have done looked to us to be the only possibility we had to test the principle.

It would be interesting to carry out experiments analogous to the above described ones for other types of systems for which reversible algorithms can be written and implemented at least in the conservative case, as discovered in [LV]: it is unclear, however, if they can be extended to cover the dissipative cases and, if so, if this can be done with the accuracy necessary to the test of the chaotic hypothesis. ${ }^{4}$

4 The important paper [LV] had escaped our attention until very recently, too late to take it into account: it proves that reversible codes do exist for systems not too far from ours and it is certainly important, and probably possible, to try to adapt them to test the chaotic hypothesis in a truly reversible CODES. 
(ii) The pairing rule, Axiom A attractors and reversibility: If the chaoticity hypothesis, in the form given in $\S 1$ for the reversible case, is interpreted as meaning that the system behaves as a transitive Anosov reversible system, with the time reversal operation being the "global time reversal" $i$, then it implies that the stable and unstable manifolds have the same dimension. So we would expect to have as many positive and negative Lyapunov exponents.

However the existence of the phenomenon in which as $E$ grows some Lyapunov exponent that is $>0$ at small $E$ becomes $<0$ at larger $E$ was already pointed out in [DPH]. And tests showed, see Fig.14 and Fig.15 below, that in the case of 10 particles with semi-periodic boundary conditions the 19-th (out of the 38) Lyapunov exponents is likely to be $<0$.

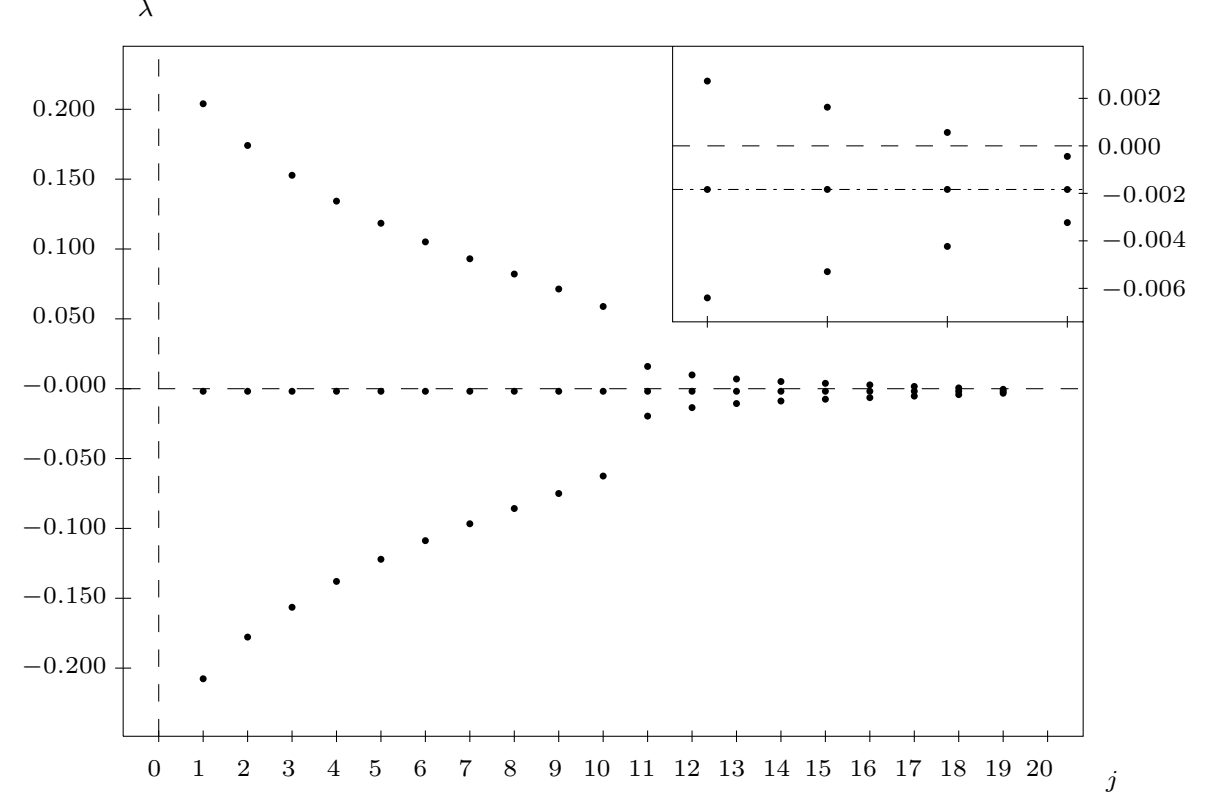

Fig.14: The 38 Lyapunov exponents for 10 and $\frac{1}{2}(p b c)$. The small picture is an enlargment of the tail of the larger one and it shows more clearly the pairing rule and that the 19-th exponent is slightly negative.

A simple test to see how reliably we have evaluated the Lyapunov exponents can be, nevertheless, easily made: it consists in testing the pairing rule, discovered in [EM], [ECM1], verified to an almost unthinkable accuracy in experiments by [DPH], and recently proved for cases that include precisely our systems, see $[\mathrm{DM}]$. Although we did not push our study to check the pairing with the remarkable precision reached in $[\mathrm{DPH}]$ we find that the pairing rule is obeyed. We stress, however, that the program we use to compute the Lyapunov exponents is very close (although not identical) to the actual scheme followed in $[\mathrm{DM}]$ to prove the pairing rule: therefore the test does not provide a test on the accuracy of the exponents. The pairing rule has in fact to be fullfilled with high precision even if the precision reached in measuring the exponents is not comparable (in other words the errors that one makes on each exponent of a pair compensate exactly, see [DM], if they are due to the shortness of the runs).

The existence of one negative exponent in excess lead us to study the question in more detail. In Fig.15 is shown the graph of the Lyapunov exponents $\lambda_{j}$, with $j=13 \div 19$ in the $\frac{1}{2} \mathrm{pbc}$ and 10 particles for $E=0.1$ to $E=5.0$ at steps of 0.1 . The results are still "raw" in the sense that we have not yet been able to make a satisfactory study of the errors: the method we used is the "usual method", [BGGS], with a test trajectory of $0.5 \cdot 10^{5}$ collisions. The errors are however quite large and more accurate experiments are necessary to confirm the raw data. 


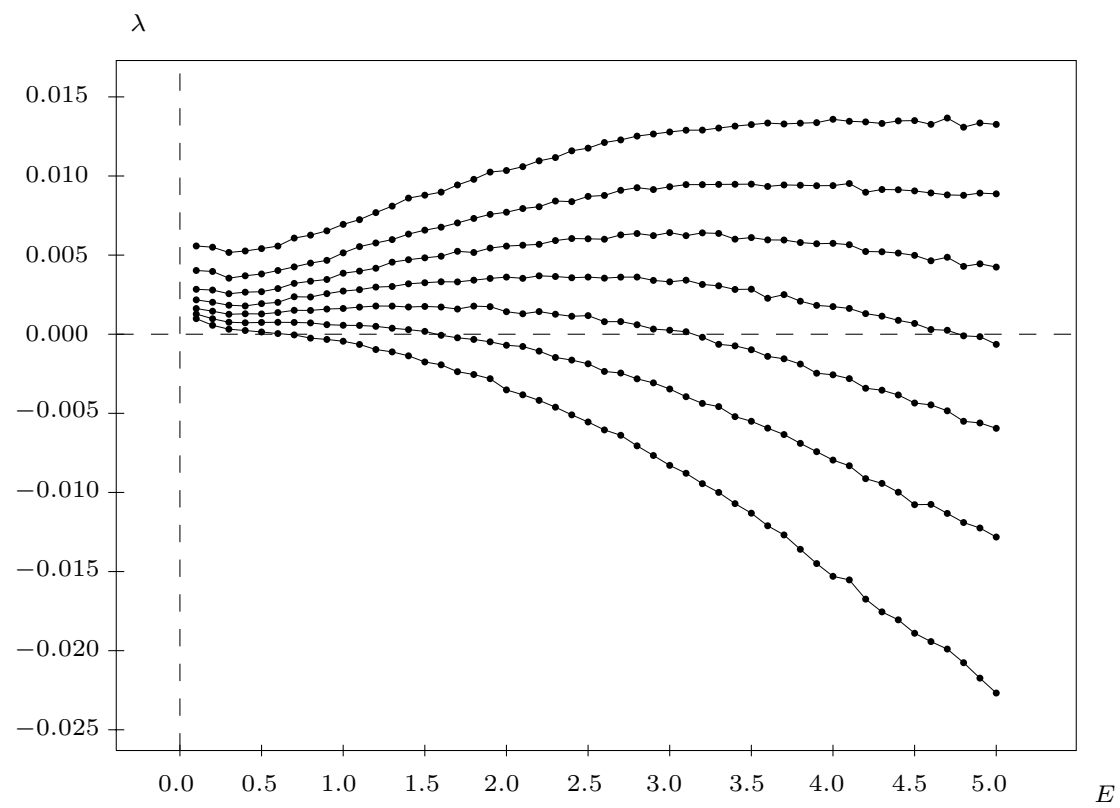

Fig.15: The 13-th through the 19-th Lyapunov exponents in the case $N=10$, $\frac{1}{2}(p b c)$ : row data (no error bars estimated). The pairing rule, [EM], [ECM1], [SEM], [DM] is verified.

The preponderance of the negative Lyapunov exponents over the positive ones does not seem due, at least in the cases where it appears clearly, to the non-reversibility of our CODES: hence the existence of more negative than positive exponents is a very likely to be a real phenomenon at large field (and fixed $N$ ).

Then one can ask the question: is there any reason to think that the fluctuation theorem might be valid even if the attractor is "only" an Axiom A attractor?

We have not been able to test the fluctuation law at values of $E$ where there was an apparent excess (we say "apparent" because our error analysis in not satisfactory, yet) of negative Lyapunov exponents, (say at $E=2.5$ in the case of 10 particles and $\frac{1}{2}(p b c)$ where we see 2 negative Lyapunov exponents in excess, see Fig.15). The reason is that the estimated time for the corresponding numerical experiment is well beyond the present day computer capacities. Large fluctuations may be very difficult to observe at very large fields.

We do not think that the fluctuation law can hold by chance: but we have seen it well verified in a situation where there seem to be one negative Lyapunov exponents in excess over the positive ones, see Fig.12 (and Fig.15 with $E=1$ ). However from the analysis in [GC1], [GC2] its validity appears very tightly related to the existence of a time reversal symmetry leaving the closure of the attractor invariant, i.e. a map $i^{*}$ defined on the closure of the attractor and such that $S i^{*}=i^{*} S^{-1}$.

Therefore we conclude that a not unreasonable scenario would be that when there are pairs of Lyapunov exponents that consist of two negative exponents then the attractor and its accumulations points can be simply regarded as a smooth lower dimensional surface. ${ }^{5}$ The motion on this lower dimensional surface (whose dimension is smaller than that of phase space by an amount equal to the number of paired negative exponents) will still have an attractor (with dimension lower than the dimension of the surface itself, as suggested by the Kaplan-Yorke formula, $[\mathrm{ER}])$. And on such manifold the motion will still be reversible in the sense that there

5 This does not preclude the possibility that the attractor has a fractal dimension (smoothness of the closure of an attractor has nothing to do with its fractal dimensionality, see [ER],[GC1],[G1]). 
will be a map $i^{*}$ of the attractor into itself (certainly different from the global time reversal map $i$ ) which inverts the time on the attractor and that can be naturally called a local time reversal, see [BG].

Then manifestly one would be back with an Anosov system (on a lower dimensional manifold) and a version of the fluctuation theorem would still hold. Furthermore one could say that this is only a different interpretation of the chaotic principle of [GC1], [GC2] (which in such case does not even require to be reformulated to apply).

If this picture is correct we can write the phase space contraction rate (see $(2.4),(2.5)) \sigma(x)=$ $\sigma_{0}(x)+\sigma_{\perp}(x)$ where $\sigma_{0}(x)$ is the contraction rate on the surface on which the attractor lies and $\sigma_{\perp}(x)$ is the contraction rate of the part of the stable manifold of the attractor which is not on the attractor itself (the angle between the part of the stable manifold sticking out of the attractor and the attractor itself is disregarded here as we think that it is bounded away from 0 and $\pi$ since the attractor is compact).

Of course the local time reversal will change the sign only of $\sigma_{0}(x)$ and the fluctuation theorem should apply to the fluctuations of $\sigma_{0}$. But $\sigma_{0}(x)$ is not directly accessible to measurement: nevertheless we can still study its fluctuations via the following heuristic analysis. From the proof in $[\mathrm{DM}]$ of the pairing rule one sees that the jacobian matrix $J$ of the map $S$ is such that $\sqrt{J^{*} J}$ has $D$ pairs of eigenvalues and the logarithms of each pair add up to $\int_{0}^{t(x)} \alpha\left(Q_{t} x\right) d t$ (see also $(2.4),(2.5))$.

The simplest interpretation of this, in view of the above proposed picture of the attractor, is that the pairs with elements of opposite signs describe expansion on the manifold on which the attractor lies. While the $M \leq D$ pairs consisting of two negative eigenvalues describe the contraction of phase space in the directions transversal to the manifold on which the attractor lies. Then we would have $t_{0} \sigma_{0}(x)=(D-M) \int_{0}^{t(x)} \alpha\left(Q_{t} x\right) d t$ and we should have a fluctuation law for the quantity $p(x)$ associated with $\sigma_{0}(x)$ defined by (2.6) and (2.7) with $\sigma_{0}$ replacing $\sigma$, i.e. (accepting the above heuristic argument):

$$
\sigma_{0 \tau}(x)=\frac{(D-M)}{D}\langle\sigma\rangle_{+} p(x)
$$

i.e. a law identical to (2.10) up to a correcting factor $1-\frac{M}{D}$ :

$$
\log \frac{\pi_{\tau}(p)}{\pi_{\tau}(-p)}=\tau t_{0}\langle\sigma\rangle_{+}\left(1-\frac{M}{D}\right) p
$$

The graphs of Fig.12 are relative to an experiment in which we see that there may be one negative exponents in excess over the positive ones (as said above, see also Fig.15): it is very small (see Fig.14), and it carries an error bar that we estimate to be so large to allow for positive values as well. The graphs, however, show that the agreement with the experiment of (6.4) is within the errors: had there been no negative exponents we would have expected a slope 1 . If there is one negative exponent in excess we expect a slope $1-\frac{1}{19}$ which is within the error bars in Fig.12 (had we drawn in Fig.12 the best fit line rather than the line with slope 1 the agreement with the slope $1-\frac{1}{19}$ would have been even better). An excess of 2 exponents would yield a slope of $1-\frac{2}{19}$ which is out of the error bars.

Note that since the exponent smallest in modulus is so small we must expect that it yields a clear effect only after extremely long times have elapsed (i.e. for values of $\tau>4.10^{3}$ : totally out of computability).

The difficulty on the above scenario is that there is no a priori reason to think that the attractors should have the above structure: i.e. fractal sets lying on smooth surfaces on phase space on which the motion is reversible. However such a picture is very suggestive and it might 
be applicable to more general situations in which the reversibility holds only on the closure of the attractor and not in the whole space (like "strongly dissipative systems"). An attempt at discussing this point can be found in [BG].

(iii) Smoothness of the closure of the attractor: The smoothness of the surface on which the attractor lies, so that the attractor can be regarded, in itself, as an Anosov system, is very likely not necessary, from a mathematical viewpoint. The more general assumption that the attractor verifies the Axiom A (and is transitive) would be sufficient if accompanied by the (very strong) assumption that the attractor is mapped into itself by a symmetry $i^{*}$ such that on the attractor $i^{*} S=S^{-1} i^{*}$ (discussed in (ii) above). We stress again that $i^{*}$ cannot be the same as the global time reversal $i$ because the latter will map the attractor for the forward motion into the one for the backward motion: however through simple examples of reversible motions with attractors closures smaller than the whole phase space (hence verifying Axiom A but not the Anosov property) one can see that the existence of $i$ often (always?) induces the existence of a map $i^{*}$ on the attractor, se [BG].

We insist in talking about smoothness for the following two reasons:

(1) because we think that the theory applies to general many particle systems and we cannot see the relevance of a possible fractional dimension over $N=10^{19}$ total dimensions: in other words we can proceed as if the dimension was integer (in [GC2] the fractality is called "an unfortunate accident" that may happen in the problems that we study), hence as if the system is Anosov, provided we accept that the attractor has a time reversal symmetry (i.e. there is a map $i^{*}$ of the attractor into itself that anticommutes with the time evolution, $S_{t} i^{*}=i^{*} S_{-t}$ ).

(2) because, as it partially appears from Fig.15, the Lyapunov exponents seem to evolve, as a function of $E$, along the following pattern. At smaller $E$ they are all non zero (as shown by Fig.15): the dimension of the closure of the attractor in $4 N-2$ (i.e. that of the phase space $\mathcal{C}$ ). Then as $E$ grows one of them crosses continuously (and with non zero $E$ derivative, i.e. transversally) the value 0 at some $E_{1}>0$ becoming negative for larger values of the field; the closure of the attractor has now dimension $4 N-4$. At $E_{2}>E_{1}$ a second Lyapunov exponent crosses 0 (transversally) and the closure of the attractor has now dimension $4 N-6$, etc. This suggests that, as $E$ varies, the attractor is characterized by more and more "constants of motion", i.e. by the vanishing of more and more observables. Every time one more "constant of motion" is born we see that the attractor loses two dimensions. Nothing suggests to us that it becomes non smooth, or appreciably so.

One should also bear in mind that the above analysis is, necessarily, carried over in systems with few degrees of freedom. It might well be that the picture can considerably simplify at large $N$. See below for some thoughts on that point.

(iv) Fluctuation theorem as a reversibility test: Since the very derivation, [GC1],[GC2],[G2], of the fluctuation theorem is so intimately related to reversibility one could say the if the predictions of the fluctuation theorem are verified, perhaps with a slope $<1$ as suggested by (6.4), then this is a sign that the dynamics on the attractor is reversible in the sense that it is mapped into itself by a map $i^{*}$ such that $i^{*} S=S^{-1} i^{*}$ (hence $i \neq i^{*}$ unless the system is Anosov and the attractor is the whole space). It is clear that the above considerations give rise to several tests that can be experimentally performed.

Note that not only the linearity in $p$ is a strong statement, but also the predicted value $<1$ is somewhat surprising: naively one could be tempted to think that the contraction rate transversal to the attractor is uniform over the attractor: this would lead to a slope $>1$ (and the larger the stronger is the attraction from the attractor).

(v) Gaussian? Central limit theorem? One may say, as a first reaction to the analysis, "of course 
we expected a Gaussian distribution of the fluctuations because the dissipation $\tau \sigma_{\tau}$ is a sum of many terms that are statistically independent" if the motion is chaotic. Hence "everybody (reasonable)" would expect a Gaussian distribution for the fluctuations. This would mean that $(p-1)$ has a dispersion of order $C \tau^{-\frac{1}{2}}$ for some (non trivial) constant $C$ related to the entropy autocorrelation function $\left\langle\sigma\left(S^{n} x\right) \sigma(x)\right\rangle$ :

$$
C^{2}=\frac{1}{\langle\sigma\rangle_{+}^{2}} \sum_{n=-\infty}^{\infty}\left(\left\langle\sigma\left(S^{n} \cdot\right) \sigma(\cdot)\right\rangle_{+}-\langle\sigma\rangle_{+}^{2}\right)
$$

And it would imply that:

$$
\log \frac{\pi_{\tau}(p)}{\pi_{\tau}(-p)}=\tau \frac{2}{C^{2}} p
$$

However this would not imply that $\frac{2}{C^{2}}=t_{0}\langle\sigma\rangle_{+}$: which would mean that, in general, it is also:

$$
\left\langle\sigma_{+}\right\rangle=\frac{t_{0}}{2} \sum_{n=-\infty}^{\infty}\left(\left\langle\sigma\left(S^{n} \cdot\right) \sigma(\cdot)\right\rangle_{+}-\langle\sigma\rangle_{+}^{2}\right)
$$

The latter would be a strange prediction in the context of the central limit theorems. Furthermore the central limit theorem is expected to hold only for fluctuations of $p-1$ the order of $\tau^{-1 / 2}$ while (2.10) compares the probabilities of $p$ to those of $-p$ which, for $p \simeq 1$, describe deviations of order $\tau$. In other words there is no relation between the usual central limit theorem for $p$ and the fluctuation theorem (when they are expected to hold, i.e. for $\tau \rightarrow \infty$ ).

Therefore it came as a surprise to us that the results were instead consistent, on the whole range of observability, with a Gaussian distribution. We would have thought that $\log \pi_{\tau}(p)=-\tau \zeta(p)$ (for $\tau$ large enough) with only the odd part of $\zeta(p)$ strictly proportional to $p$, while the even part had no reason to be quadratic. And the example of $\S 3$ shows how natural it would be that is not quadratic. The constant $C$ would have been mainly determined by the curvature of $\zeta(p)$ at its maximum $p=1$, totally unrelated (we thought) to $\frac{1}{2} t_{0}\langle\sigma\rangle_{+}$.

Naturally we expected (6.7) to hold for very small external field $E$ : in the case $N=1$ it was indeed proved by [CELS] to hold for values of $E$ that, in our units, are extremely small compared to 1: and the equation (6.7) is known for small field ("linear regime") as the "fluctuation dissipation" relation (or "Green-Kubo formula"). This relation has been shown to be closely related (and essentially a consequence) of the chaotic hypothesis in reversible systems for $E$ close to 0, (see [G5] eq. (5.9), taking into account the special form of $\sigma$, its linearity in $E$ and the fact that the energy is 1) still with corrections of $O\left(E^{3}\right)$.

Note that as $E \rightarrow 0$ the two sides of (6.7) have both size of order $O\left(E^{2}\right)$ and the fluctuation dissipation theorem is the relation obtained by dividing the two sides by $E^{2}$ and letting $E \rightarrow 0$. There should be a deeper reason for the relation between the small deviations constant $C$ (relevant in the fluctuations of scale $\sqrt{\tau}$ ) and the large scale fluctuations (of size $O(\tau)$ ) which are related to $\langle\sigma\rangle_{+}$, see (2.10): this seems to be explained in [G6].

In the "old" literature one can find statements that today sound somewhat mysterious like:

"It is empirically known that for macroscopic values of $\underline{\alpha}$, i.e. for values of the $\alpha_{i}$ much larger than their root mean square values at equilibrium, the averages of these quantities frequently obey linear differential equations."

which is then used to establish that relations between properties that hold for small fluctuations hold also for large ones, at least in the average. In [DGM], p. 100, the above statement is the beginning of a classical derivation of the Onsager reciprocity relations. One may speculate, 
since the chaotic hypothesis is related to the Onsager relations as well, [G5], that our "strange" relation between the small and large deviations might be related to the fact that a similar property can be taken as the basis of a derivation of Onsager's theory of reciprocity. Small and large fluctuations seem to have some common properties that one may not expect a priori or, at least, that one may consider worth of being challenged and tested, see [DGM], p. 102. This point of view leads to the paper [G6] where it is developed and its consequences are drawn.

The connection between large and small fluctuations is, if at all present, likely to be a peculiarity of models typical of statistical mechanics (short range interacting many particle systems): it may not hold in other cases in which the chaotic hypothesis can be applied (sometimes leading, nevertheless, to reciprocity relations of Onsager type) like in fluidodynamics models, [G4].

It is worth stressing again that we know that the distribution in $p$ cannot be Gaussian for all $p$ 's: because there is a maximum ( $\tau$-independent) value that $p$ can take, just from the finiteness of phase space. This value, called $p^{*}$ in [G2], can be easily measured in our experiments (see (2.12)) but it is very far away from the region where we have enough statistics to make meaningful measurements, as it appears from the graphs reported above.

(vi) Time scales for large $N$ : A somewhat more speculative scenario can be drawn for large $N$. We mention it because we hope to receive some help in a program directed to test it. It seems reasonable to us that in the thermodynamic limit the Lyapunov exponents of the system will fall into two categories each consisting of a pair of exponents. The sum of the values of each pair is constant and equal to half the average entropy creation rate (this is the pairing rule, see above).

A large number of pairs $(O(N))$ will consist of one vanishing exponent and its negative companion. The remaining positive Lyapunov exponents will all be identical: marking the time scale of local approach to equilibrium, hence also the other negative exponents will be identical (by the pairing rule). By identical we mean here that the ratio between the largest and the smallest positive Lyapunov exponents is bounded uniformly in $N$ (away from 0 and $\infty$ ).

This is in perfect agreement with fig. 5 of $[\mathrm{DPH}]$ describing a very high density system: it is not in agreement with the other results of [DPH].

Nevertheless, as argued in also in [G3], it is possible that the low density results are flawed in this respect as one would need far too large systems to obtain Lyapunov exponents for a distribution close to the thermodynamic limit distribution $\mu$. Only in the high density case a small sample of gas exhibits the features of a large sample.

The above picture merges with the ideas in [G4] relating the 0 Lyapunov exponents to the macroscopic modes described by macroscopic equations, while the nonzero exponents describe the approach to local equilibrium. It also matches with Fig.14 where a rather sharp drop of the Lyapunov exponents towards 0 appears betwen the 10-th and the 11-th exponent.

Since it is always assumed that there is only one microscopic time scale for the local approach to equilibrium it is perhaps a natural conjecture that the Lyapunov exponents should have the above structure.

(vii) We see that the above experiments raise perhaps more problems than expected: but the chaotic hypothesis emerges as not inconsistent with the data.

\section{$\S$ Appendix. Fits and Errors.}

This appendix is adapted to the present experiments from the corresponding appendix in [GG]. We get data sets from our computer experiments, say $\underline{y}(\underline{x})=\left\{y\left(x_{i}\right)\right\}_{i=1, N}$, where $\underline{x}=\left\{x_{i}\right\}_{i=1, N}$ is in our case an independent variable, say for instance a set of $N$ collision numbers or time instants. To the latter experimental data set of points, we want to fit a given guessed function, say $f(x ; \underline{\alpha})$, where $\underline{\alpha}=\left\{\alpha_{n}\right\}_{n=1, p}$ is a set of arbitrary parameters. Here by 
fit we mean to find a set of parameters $\underline{\alpha}^{*}$ which optimizes some reasonable functional relation between the experimental data and the fitting function.

In our case we use the least squares functional, i.e. :

$$
V(\underline{y}(x), \underline{\alpha})=\sum_{i=1}^{N}\left[y_{i}-f\left(x_{i} ; \underline{\alpha}\right)\right]^{2}
$$

The set of parameters $\underline{\alpha}^{*}(\underline{y})$ is here obtained by asking that they should be the minima of the $V$ function: $\partial_{\underline{\alpha}} * V\left(\underline{y}, \underline{\alpha}^{*}\right)=0$. We also define the goodness of our fit, $G$, by the average $y$-distance of our data to the function $f\left(x ; \underline{\alpha}^{*}\right): G(\underline{y}(\underline{x}))=\left(V\left(\underline{y}(\underline{x}), \underline{\alpha}^{*}\right) / N\right)^{1 / 2}$. This parameter is only meaningful when it is compared with the one from another fit. Given many fits, the one with smallest $G$ value will be called best fit (among the considered fits).

The data have, in general, non-negligible errors, say $\underline{\varepsilon}=\left\{\varepsilon_{i}\right\}_{i=1, N}$, due to the finite number of samples used in the averaging (see comments in $\S 2$ ). Such errors induce errors on the parameter values. Therefore, a measure of the error amplitude in $\underline{\alpha}^{*}(\underline{y})$ is given by:

$$
\Delta_{\underline{\varepsilon}} \underline{\alpha}^{*}(\underline{y})=\left[\underline{\alpha}^{*}(\underline{y}+\underline{\varepsilon})-\underline{\alpha}^{*}(\underline{y}-\underline{\varepsilon})\right] / 2
$$

In the particular case in which the magnitude of the data error is much smaller than the measured value, $\left|\varepsilon_{i} / y\left(x_{i}\right)\right|<<1$, we may expand the latter equation around $\underline{\varepsilon}=\underline{0}$ :

$$
\Delta_{\underline{\varepsilon}} \alpha_{n}(\underline{y})=\sum_{i=1}^{N} c_{i}^{(n)}(\underline{y}) \varepsilon_{i}
$$

The coefficients $c_{i}^{(n)}$ are found by expanding $V(\underline{y}(\underline{x})+\underline{\varepsilon}, \underline{\alpha})$ around $\underline{\alpha}^{*}(\underline{y})$ and $\underline{\varepsilon}=\underline{0}$ and they are given by:

$$
c_{i}^{(n)}=-\sum_{m=1}^{p} D_{m n}^{-1} \partial_{y\left(x_{i}\right) \alpha_{m}^{*}}^{2} V\left(\underline{y}, \underline{\alpha}^{*}\right)
$$

where $D_{m n}=\partial_{\alpha_{m}^{*} \alpha_{n}^{*}}^{2} V\left(\underline{y}, \underline{\alpha}^{*}\right)$.

In particular for the linear fit, $f(x, \underline{\alpha})=\alpha_{1}+\alpha_{2} x$, the coefficients $c_{i}^{(1),(2)}$ are given by:

$$
c_{i}^{(1)}=\frac{2}{N \Delta x}\left(\overline{x^{2}}-\bar{x} x_{i}\right), \quad c_{i}^{(2)}=\frac{2}{N \Delta x}\left(x_{i}-\bar{x}\right)
$$

where $\Delta x=\overline{(x-\bar{x})^{2}}$ and $\overline{x^{n}}=N^{-1} \sum_{i=1}^{N} x_{i}^{n}$.

The errors are random variables and we have to average them over their distribution. In all cases considered it seemed reasonable to consider the errors $\varepsilon_{i}$ as independent variables. Therefore we empirically estimate an upper bound for their correlation values:

$$
\left|\left\langle\varepsilon_{i} \varepsilon_{j}\right\rangle\right| \leq \sqrt{\left\langle\varepsilon_{i}^{2}\right\rangle\left\langle\varepsilon_{j}^{2}\right\rangle}
$$

The parameter errors in our analysis are defined by the equations:

$$
\Delta \alpha_{n}(\underline{y})^{2}=\sum_{i=1}^{N} \sum_{j=1}^{N} c_{i}^{(n)} c_{j}^{(n)} \delta_{i} \delta_{j} \geq\left\langle\Delta_{\underline{\varepsilon}} \alpha_{n}^{2}\right\rangle
$$

where $\delta_{i}^{2}=\left\langle\varepsilon_{i}^{2}\right\rangle$ and their use and meaning is described entirely by the above comments. 
Acknowledgments: We are indebted to E.G.D. Cohen for his interest and his comments and suggestions: his constant support and advice have been very important to us. We thank J. Lebowitz and D. Ruelle for many discussions during which they provided inspiration and ideas. This work is part of the research program of the European Network on: "Stability and Universality in Classical Mechanics", \# ERBCHRXCT940460, and it has also been partly supported by Rutgers University, CNR-GNFM, IHES and MPI.

\section{References}

[AA] Arnold, V., Avez, A.: Ergodic problems of classical mechanics, Benjamin, 1966.

[Bo] Bowen, R.: Equilibrium states and the ergodic theory of Anosov diffeomorphisms, Lecture notes in mathematics, vol. 470, Springer Verlag, 1975.

[BG] Bonetto, F., Gallavotti, G.: Reversibility, coarse graining and the chaoticity principle, in preparation.

[BEC] Baranyai, A., Evans, D.T., Cohen, E.G.D.: Field dependent conductivity and diffusion in a two dimensional Lorentz gas, Journal of Statistical Physics, 70, 1085-1098, 1993.

[BGGS] Benettin, G., Galgani, L., Giorgilli, A., Strelcyn, J.M.: Lyapunov Characteristic Exponents for Smooth Dynamical Systems and for Hamiltonian Systems; a Method for Computing all of Them. Part 1: Theory, Meccanica, 15, 9-20, 1980; and Lyapunov Characteristic Exponents for Smooth Dynamical Systems and for Hamiltonian Systems; a Method for Computing all of Them. Part 2: Numerical Applications. Meccanica 15, 21-30 (1980).

[CELS] Chernov, N.I., Eyink, G.L., Lebowitz, J.L., Sinai, Y.G.: Steady state electric conductivity in the periodic Lorentz gas, Communications in Mathematical Physics, 154, 569-601, 1993.

[DM] Dettman, C.P., Morriss, G.P.: Proof of conjugate pairing for an isokinetic thermostat, N.S. Wales U., Sydney 2052, preprint, 1995.

[DPH] Dellago, C., Posch, H., Hoover, W.: Lyapunov instability in system of hard disks in equilibrium and non-equilibrium steady states, preprint, Institut für Experimentalphysik, Universität Wien, Austria, August 1995.

[DGM] de Groot, S., Mazur, P.: Non equilibrium thermodynamics, Dover, 1984.

[EM] Evans, D.J., Morriss, G.P.: Statistical mechanics of nonequilibrium liquids, Academic Press, 1990.

[ECM1] Evans, D.J.,Cohen, E.G.D., Morriss, G.P.: Viscosity of a simple fluid from its maximal Lyapunov exponents, Physical Review, 42A, 5990-5997, 1990.

[ECM2] Evans, D.J.,Cohen, E.G.D., Morriss, G.P.: Probability of second law violations in shearing steady flows, Physical Review Letters, 71, 2401-2404, 1993.

[ER] J. Eckmann, D. Ruelle: Ergodic theory of strange attractor, Reviews of Modern Physics, 57, $617-656,1993$

[G1] Gallavotti, G.: Topics in chaotic dynamics, Lectures at the Granada school 1994, Ed. P. Garrido, J. Marro, in Lecture Notes in Physics, Springer Verlag, 448, p. 271-311, 1995.

[G2] Gallavotti, G.: Reversible Anosov diffeomorphisms and large deviations., Mathematical Physics Electronic Journal, 1, (1), 1995.

[G3] Gallavotti, G.: Coarse graining and chaos, in preparation.

[G4] Gallavotti, G.: Chaotic principle: some applications to developed turbulence, in mp_arc @math. utexas. edu, \#95-232, 1995.

[G5] Gallavotti, G.: Chaotic hypothesis: Onsager reciprocity and fluctuation-dissipation theorem, in print on J. Statistical Physics.

[G6] Gallavotti, G.: Extension of Onsager's reciprocity to large fields and the chaotic hypothesis, mp_ arc 96-109; or chao-dyn 9603003.

[GC1] Gallavotti, G., Cohen, E.G.D.: Dynamical ensembles in nonequilibrium statistical mechanics, Physical Review Letters, 74, 2694-2697, 1995. 
[GC2] Gallavotti, G., Cohen, E.G.D.: Dynamical ensembles in stationary states, Journal of Statistical Physics, 80, 931-970, 1995.

[GG] Garrido, P., Gallavotti, G.: Billiards correlation functions, Journal of Statistical Physics, 76, 549-586, 1994.

[HHP] Holian, B.L., Hoover, W.G., Posch. H.A.: Resolution of Loschmidt's paradox: the origin of irreversible behavior in reversible atomistic dynamics, Physical Review Letters, 59, 10-13, 1987.

[L] Lorenz, E.: A deterministic non periodic flow, Journal of Atmospheric Science, 20, 130- 141, 1963.

[LA] Levi-Civita, T., Amaldi, U.: Lezioni di Meccanica Razionale, Zanichelli, Bologna, 1927 (reprinted 1974), volumes $I, I I_{1}, I I_{2}$.

[LV] Levesque, D., Verlet, L.: Molecular dynamics and time reversibility, Journal of Statistical Physics, 72, 519-537, 1993.

[M] Morriss, G.: , Physics Letters, 134A, 307, 1989.

[PH] Posch, H.A., Hoover, W.G.: Non equilibrium molecular dynamics of a classical fluid, in "Molecular Liquids: New Perspectives in Physics and Chemistry", ed. J. Teixeira-Dias, Kluwer Academic Publishers, p. 527-547, 1992.

[R1] Ruelle, D.: Chaotic motions and strange attractors, Lezioni Lincee, notes by S. Isola, Accademia Nazionale dei Lincei, Cambridge University Press, 1989; see also: Ruelle, D.: Measures describing a turbulent flow, Annals of the New York Academy of Sciences, 357, 1-9, 1980. For more technical expositions see Ruelle, D.: Ergodic theory of differentiable dynamical systems, Publications Mathémathiques de l' IHES, 50, 275-306, 1980.

[R2] Ruelle, D.: A measure associated with Axiom A attractors, American Journal of Mathematics, 98, 619-654, 1976.

[R3] Ruelle, D.: Positivity of entropy production in non equilibrium statistical mechanics, IHES preprint, 1995.

[S] Sinai, Y.G.: Gibbs measures in ergodic theory, Russian Mathematical Surveys, 27, 21-69, 1972. Also: Lectures in ergodic theory, Princeton U. Press, Princeton, 1977.

[Sm] Smale, S.: Differentiable dynamical systems, Bullettin of the American Mathematical Society, 73 , 747-818, 1967.

[SEM] Sarman, S., Evans, D.J., Morriss, G.P.: Conjugate pairing rule and thermal transport coefficients, Physical Review, 42A, 2233-2242, 1992.

[UF] Uhlenbeck, G.E., Ford, G.W.: Lectures in Statistical Mechanics, American Mathematical society, Providence, R.I., pp. 5,16,30, 1963.

Internet access: All the Authors' quoted preprints can be found and freely downloaded (latest postscript version including corrections of misprints and errors) at:

http://chimera.roma1.infn.it

in the Mathematical Physics Preprints page. 